\title{
Distribución de la precipitación y la evapotranspiración en territorio argentino de Cuenca del Plata
}

\section{Distribution of precipitation and evapotranspiration on the Argentine territory of the La Plata Basin}

HÄMMERLY, R. ${ }^{1}$; PAOLI, C. $1,{ }^{2}$ \& DUARTE,O.C. ${ }^{1,3}$

(1) Facultad de Ingeniería y Ciencias Hídricas de la Universidad Nacional del Litoral, Ciudad Universitaria, 3000 Santa Fe, Argentina (e-mail: rhammer@fich.unl.edu.ar)

(2) Centro Regional Litoral del Instituto Nacional del Agua, Argentina

(3) Facultad de Ciencias Agropecuarias de la Universidad Nacional de Entre Ríos, Entre Ríos, Argentina

https://doi.org/10.17979/cadlaxe.2018.40.0.4913

enviado 2/1/2018 aceptado 12/12/2018

\begin{abstract}
Land management and land use planning need a sound knowledge of the available natural resources, and water is one of the most important of these resources. Accurate determination of the water balance and available water for different uses requires a rather dense network of hydrometeorological measurements. Precipitation (P) and potential evapotranspiration (ETP) are two terms of the water balance of major research interest in several disciplines, including Hydrology, Climatology and Agronomy. Meteorological station networks provide P and ETP data, which are commonly used for interpolation. This study analyses the spatial and temporal distribution of P and ETP on the Argentina territory of the La Plata basin. The
\end{abstract}


analysis covers the 1970-2010 period and the data sets studied were provided by the National Water Resources Authority (SSRH), National Meteorological Service (SMN) and National Agricultural Technology Institute (INTA). The study was conducted both, at the annual and monthly temporal scales. Missing data were estimated using conventional methods. Pluviometry was available for 94 locations, while ETP was estimated from meteorological data at 38 weather stations using the Penman-Monteith method. The results showed specific trends for the spatial and temporal distribution of P and ETP. Average annual rainfall ranged from 499 $\mathrm{mm}$ to $2077 \mathrm{~mm}$. Most of the studied area of the La Plata basin in Argentina is lowlands or plains, where $\mathrm{P}$ shows a strong trend to increase from east to west; however to the west of the plains, in Andes region, P may increase with altitude. Average annual ETP ranged from 899 $\mathrm{mm}$ to $1719 \mathrm{~mm}$. Roughly, ETP decreases as P increases, even if there is not a total overlap of maxima and minima values of these two variables. Subsequently, the Chaco Seco region located to west of the plains ETP becomes higher than P. Analysis of temporal variability of yearly rainfall in the period 1970-2010 showed a clear increasing trend from 1970s to 2000, followed by a modest decreasing trend between 2000 and 2010. The information obtained in this study is highly important for water resource management and allocation planning, hydrological modelling, recharge assessment, and irrigation scheduling.

Key words: La Plata Basin, Argentina, rainfall, evapotranspiration, spatial and temporal variability

\section{Resumen}

La planificación y gestión del territorio necesitan un acabado conocimiento de la disponibilidad de los recursos naturales, y el agua es uno de los más importantes. La determinación precisa del balance de agua y el agua disponible para diferentes usos requiere de una red bastante densa de mediciones hidrometeorológicas. La precipitación $(\mathrm{P})$ y la evapotranspiración potencial (ETP) son dos términos del balance hídrico de mayor interés en varias disciplinas, incluida la hidrología, la climatología y la agronomía. Las redes de estaciones meteorológicas proporcionan datos de $\mathrm{P}$ y ETP, que son comúnmente usadas para la interpolación. Este trabajo analiza la distribución espacial y temporal de P y ETP en el territorio argentino de Cuenca del Plata. El análisis abarca el período 1970-2010 cuyos datos fueron proporcionados por el Servicio Meteorológico Nacional (SMN), la Subsecretaría de Recursos Hídricos de la Nación (SSRH) y el Instituto Nacional de Tecnología Agropecuaria (INTA). El estudio se realizó tanto a escala anual como mensual. Los datos faltantes se estimaron utilizando métodos convencionales. La pluviometría estaba disponible para 94 estaciones, mientras que la ETP se estimó a partir de datos meteorológicos en 38 estaciones, utilizando el método de Penman-Monteith. Los resultados mostraron tendencias para la distribución espacial y temporal de P y ETP. La precipitación media anual osciló entre 499 mm y 2077 mm. La mayor parte del área estudiada de la cuenca del Plata en Argentina es de planicie o llanuras, donde P muestra una fuerte tendencia a aumentar de este a oeste; sin embargo, al oeste de las llanuras, en la región de los Andes, $\mathrm{P}$ aumenta con la altitud. El promedio anual de ETP osciló entre $899 \mathrm{~mm}$ y $1719 \mathrm{~mm}$. Generalmente, la ETP disminuye a medida que P aumenta, incluso 
si no hay una superposición total de los valores máximos y mínimos de estas dos variables. Posteriormente, en la región de Chaco Seco ubicada al oeste de la planicie, la ETP se vuelve más alta que $\mathrm{P}$. El análisis de la variabilidad temporal de la precipitación anual en el período 1970-2010 mostró una clara tendencia creciente desde 1970 hasta 2000, seguida de una modesta tendencia decreciente entre 2000 y 2010. La información obtenida en este trabajo es muy importante para la gestión de los recursos hídricos y la planificación en la asignación del recurso, el modelado hidrológico, la evaluación de la recarga y la programación del riego.

Palabras Claves: Cuenca del Plata, Precipitación, Evapotranspiración, Variabilidad espacial y temporal 



\section{INTRODUCCIÓN}

Los procesos básicos que incluye el ciclo hidrológico son: precipitación evapotranspiración, infiltración, percolación y escorrentía. La precipitación se genera en la atmósfera por condensación del vapor de agua; ocurre cuando las nubes, alcanzan un punto de saturación. La evapotranspiración, puede ser considerada, como el proceso combinado de evaporación desde superficies líquidas y de transpiración o vaporización del agua líquida de los tejidos de las plantas. Tanto la evaporación como la transpiración varían según la radiación, la temperatura, la humedad atmosférica y la velocidad del viento. El término evapotranspiración potencial (ETP) se refiere a la cantidad de agua que podría pasar a la atmósfera si las disponibilidades de agua fuesen ilimitadas. Por tanto, la ETP se define como la tasa máxima de transferencia de agua desde la cubierta vegetal, superficies libres de agua, suelo y vegetación en unas condiciones óptimas de suministro, con el suelo y vegetación existente. La ETP se diferencia de la evapotranspiración real (ETR) en que en esta última se tienen en cuenta las disponibilidades de agua.

La ETP es una componente del balance de agua y un parámetro importante en la definición de las necesidades hídricas de los cultivos, por lo tanto tiene una importancia hidrológica y agronómica. En los procesos de evapotranspiración existen dos medios intercambiantes agua y aire, y muchos de los factores que influyen en el proceso están relacionados entre sí, ésta dependencia dificulta la tarea de analizarlos independientemente. Pueden ser agrupados en dos categorías según sean propios de la atmósfera ambiente en la vecindad de la superficie evaporante o referidos a la superficie evaporante pro- piamente dicha. Cuando nos referimos a la ETP, nos independizamos de considerar la naturaleza y el estado de la superficie evaporante, sin embargo la estimación de la evapotranspiración potencial sigue resultando compleja debido a que se determina por una serie de variables meteorológicas de difícil evaluación por su efecto relativo como: radiación solar, déficit higrométrico, temperatura del aire, insolación, velocidad del viento y presión barométrica.

Partiendo del conocimiento de la precipitación y de la evapotranspiración mensual estimada, se puede estudiar y modelizar los caudales en el cierre de cuencas, o en secciones de control intermedias, y el balance del agua en el suelo a lo largo del año. Por tanto, los datos de precipitación y ETP son necesarios para definir la falta y excesos de agua y encuentra aplicación, entre otros, para caracterizar la hidrología de una zona, la planificación hidráulica, la evaluación del consumo de agua y las necesidades de riego de los cultivos y las clasificaciones climática. Hay que destacar además que la precipitación y la ETP son datos de entrada para la modelización en disciplinas como la Agronomía, la Hidrología la Climatología y la Conservación de suelos. Ejemplo de ellos son en Agronomía ISAREG (PEREIRA et al., 2003) y en Hidrología HEC-HMS (Hydrologic Modeling System, USACE, 2000) y SWAT (Soil and Water Assessment Tool, ARNOLD et al., 1993); CHAC (Cálculo Hidrometeorológico de Aportaciones y Crecidas, CEDEX, 2003a), entre otros.

En definitiva, actualmente para planificar y gestionar el territorio se requiere del conocimiento acabado de los recursos disponibles, siendo el agua uno de los más importantes. Para que las demandas de agua sean satisfechas se requiere evaluar la asig- 
nación del recurso. Es por lo tanto necesario contar con la información para conocer los volúmenes de agua disponibles para ser distribuidos en el tiempo y en el espacio, esta información se refiere tanto a la precipitación como a la evapotranspiración, consideradas como variables de entradas y salidas de las cuencas en los periodos considerados.

La Cuenca del Plata es, sin dudas, la más importante unidad geográfica Argentina, en términos estratégicos, económicos, sociales, administrativos y políticos. Es por ello que una adecuada gestión desde el conocimiento de la oferta hídrica redundará en beneficios de productividad y desarrollo junto con premisas de equidad social y sustentabilidad ambiental y eficiencia económica (HÄMMERLY et al., 2014).

Dicha cuenca es la quinta más grande del mundo y la segunda en importancia en América de Sur. Es compartida por cinco países: gran parte de Brasil; el suroeste de Bolivia; el oeste de Uruguay; todo el Paraguay y el norte y centro-este de Argentina, con regiones como la Mesopotamia, el Chaco (Húmedo y Seco) y la Pampa Húmeda, incluyendo también la Puna. Esto nos da una idea de las características bien diferenciadas de las subcuencas.

Tiene una superficie aproximada de $3.100 .000 \mathrm{~km}^{2}$, equivalente al $17 \%$ de la superficie de América del Sur. La mayor parte de esta área de drenaje se encuentra en Brasil, donde alcanza $1.415 .000 \mathrm{~km}^{2}$, luego le sigue la Argentina con $920.000 \mathrm{~km}^{2}$, Paraguay, $410.000 \mathrm{~km}^{2}$, Bolivia, $205.000 \mathrm{~km}^{2} \mathrm{y}$ Uruguay $150.000 \mathrm{~km}^{2}$ (PAOLI \& SCHREIDER, 2000).

En su hidrografía se encuentran tres grandes sistemas: el Paraná, el Paraguay y el Uruguay, con superficies de $1.510 .000 \mathrm{~km}^{2}$, $1.095 .000 \mathrm{~km}^{2}$ y $365.000 \mathrm{~km}^{2}$ respectivamen- te, correspondiendo los restantes 130.000 $\mathrm{km}^{2}$ a la cuenca propia del río de la Plata. El Paraná y el Uruguay concurren a formar el río de la Plata, mientras que el Paraguay tributa directamente al Paraná. Por sus cauces escurren importantes volúmenes de agua, con caudales módulos del rio Paraná en Posadas del orden de los $14.000 \mathrm{~m}^{3} / \mathrm{s}$, luego recibe los aportes del río Paraguay de unos $5.000 \mathrm{~m}^{3} / \mathrm{s}$, lo que resultan unos $19.000 \mathrm{~m}^{3} / \mathrm{s}$ en la sección de Corrientes, para descargar en el río de la Plata un caudal de $19.700 \mathrm{~m}^{3} / \mathrm{s}$ que junto al caudal del río Uruguay de 7.000 $\mathrm{m}^{3} / \mathrm{s}$ y los aportes propios del río de la Plata conforman un caudal total de $27.000 \mathrm{~m}^{3} / \mathrm{s}$. Los caudales específicos varían entre $1 \mathrm{l} / \mathrm{s} /$ $\mathrm{km}^{2}$ hasta $21 \mathrm{1} / \mathrm{s} / \mathrm{km}^{2}$, mostrando la variabilidad regional de la cuenca (HÄMMERLY et al., 2016). El régimen de estos grandes ríos, difiere sustancialmente del de sus afluentes, no sólo por la magnitud, sino también por sus características propias, por lo tanto es conveniente la evaluación regional de las disponibilidades de agua.

Se estima que aproximadamente el $70 \%$ del Producto Bruto Interno (PBI) de los países que comparten la Cuenca del Plata es generado por actividades económicas realizadas dentro de la misma. Entre las actividades económicas se encuentra gran parte de la producción industrial y agrícola de los países que componen la cuenca. Además, en términos de generación de energía hidroeléctrica, es la segunda más importante del mundo, quedando atrás sólo de la cuenca del río Yangtze, en China. Cuatro de los cinco países que la componen: Argentina, Brasil, Paraguay y Uruguay, dependen fuertemente de la energía hidroeléctrica producida en ríos como el Paraná, el Uruguay y sus afluentes (COLLISCHONN et al., 2015). 


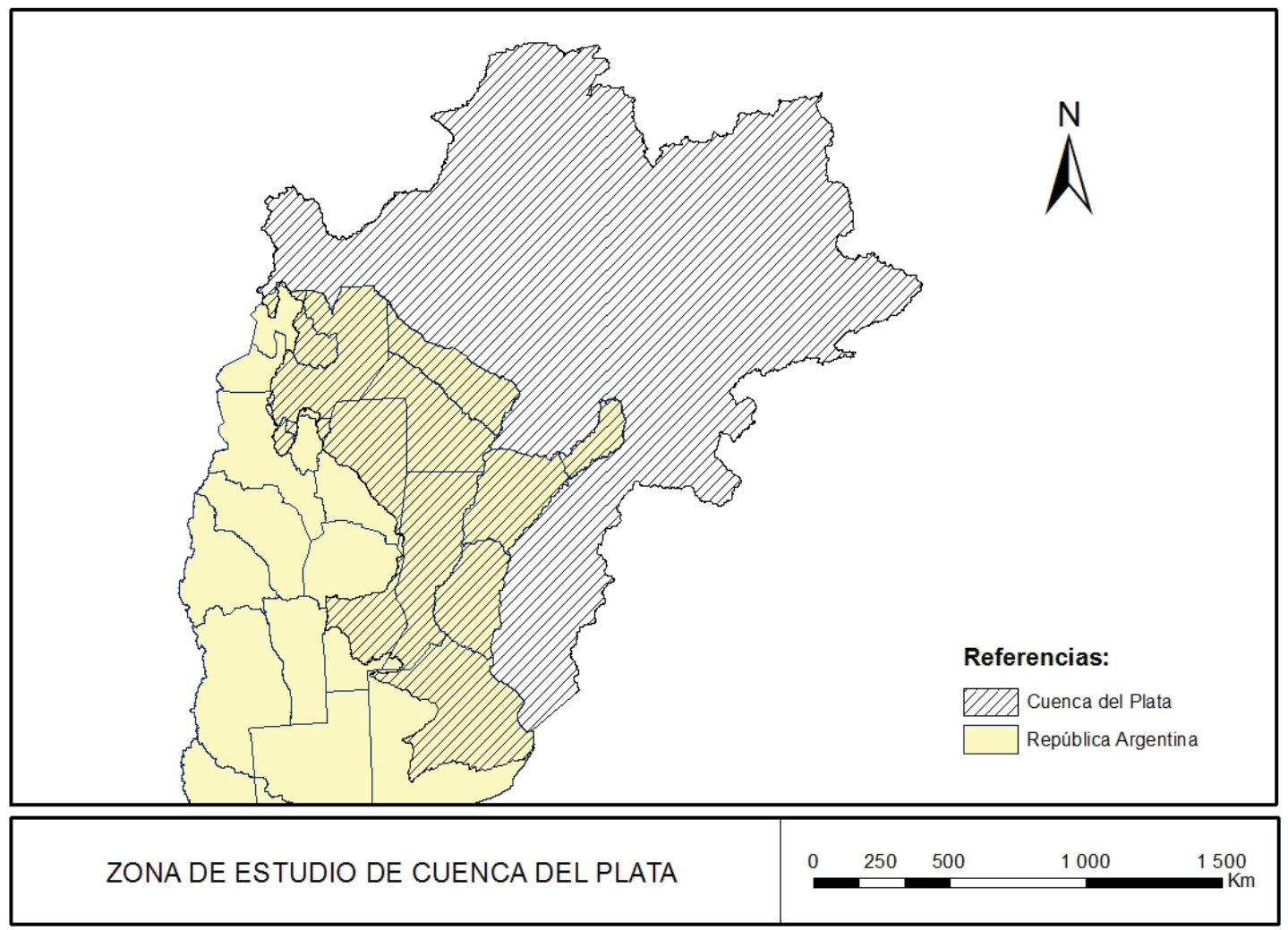

Fig. 1. Cuenca del Plata en la República Argentina y países limítrofes.

Otra actividad económica realizada en la cuenca, que es fundamental para los países de la región, y está muy relacionada con los recursos hídricos, es el transporte por vía fluvial. El río de la Plata, el río Paraná, el río Paraguay y algunos de sus afluentes se utilizan intensamente para el transporte de bienes producidos dentro y en el entorno de la cuenca. La Figura 1 muestra el área correspondiente a la porción Argentina en Cuenca del Plata.

El objetivo de este trabajo es el análisis de la distribución espacial y temporal de la precipitación y la evapotranspiración potencial en el territorio argentino de cuenca del Plata, lo que permite disponer de información confiable para ser utilizada en modelos de estimación de caudales, e incluso como línea de base para el análisis de escenarios futuros de cambio climático.

\section{MATERIAL Y MÉTODOS}

Se analizaron series de datos mensuales de precipitación y de variables meteorológicas empleadas para el cálculo de la ETP, siendo el período de análisis de 40 años (1970-2010). Estas series son lo más completas posibles y confiables, y proceden de tres organismos oficiales, y de reconocido prestigio en la adquisición de datos, de la República Argentina, como se describe a continuación

\section{Fuentes de información}

Se utilizaron datos climáticos de estaciones que pertenecen a la Subsecretaria de Recursos Hídricos de la Nación (SSRH), cuya localización se puede observar en la Figura 2, el Servicio Meteorológico Nacional (SMN), 
presentadas en la Figura 3 y el Instituto Nacional de Tecnología Agropecuaria (INTA), presentadas en la Figura 4, a paso de tiempo mensual. Algunas de las estaciones posean registros sólo de precipitación, mientras que en otras se miden variables climáticas como la temperatura, la humedad relativa, las horas de sol o la velocidad del viento, a partir de las cuales se puede calcular la ETP.

El número total de estaciones pluviométricas utilizadas ha sido de 94 de las cuales 51 pertenecen a la SSRH, 25 al Servicio Meteorológico Nacional y 18 al Instituto Nacional de Tecnología Agropecuaria. Resultando 13 estaciones con series completas del SMN y 11 del INTA, mientras que la mayoría de los datos pluviométricos de las estaciones que pertenecen a la SSRH comienza a partir de la década del 80 (HÄMMERLY, 2012).
El número de estaciones que han registrado otras variables meteorológicas es inferior y asciende a 38. La Subsecretaria de Recursos Hídricos de la Nación dispone solamente de 5 que finalmente no se usaron, mientras que el SMN aporta 21 de las cuales 8 poseen registros completos y al INTA le corresponden 17 de las cuales 11 no tienen faltantes. Las Figuras 2 a 4 muestran la ubicación de las estaciones de las tres fuentes.

Aunque la información analizada se refiere al período global 1970 al 2010, las diferentes estaciones y variables medidas corresponden a distintas fechas de inicio y finalización, y a la vez presentan interrupciones que han sido volcadas en gráficos de disposición de información.

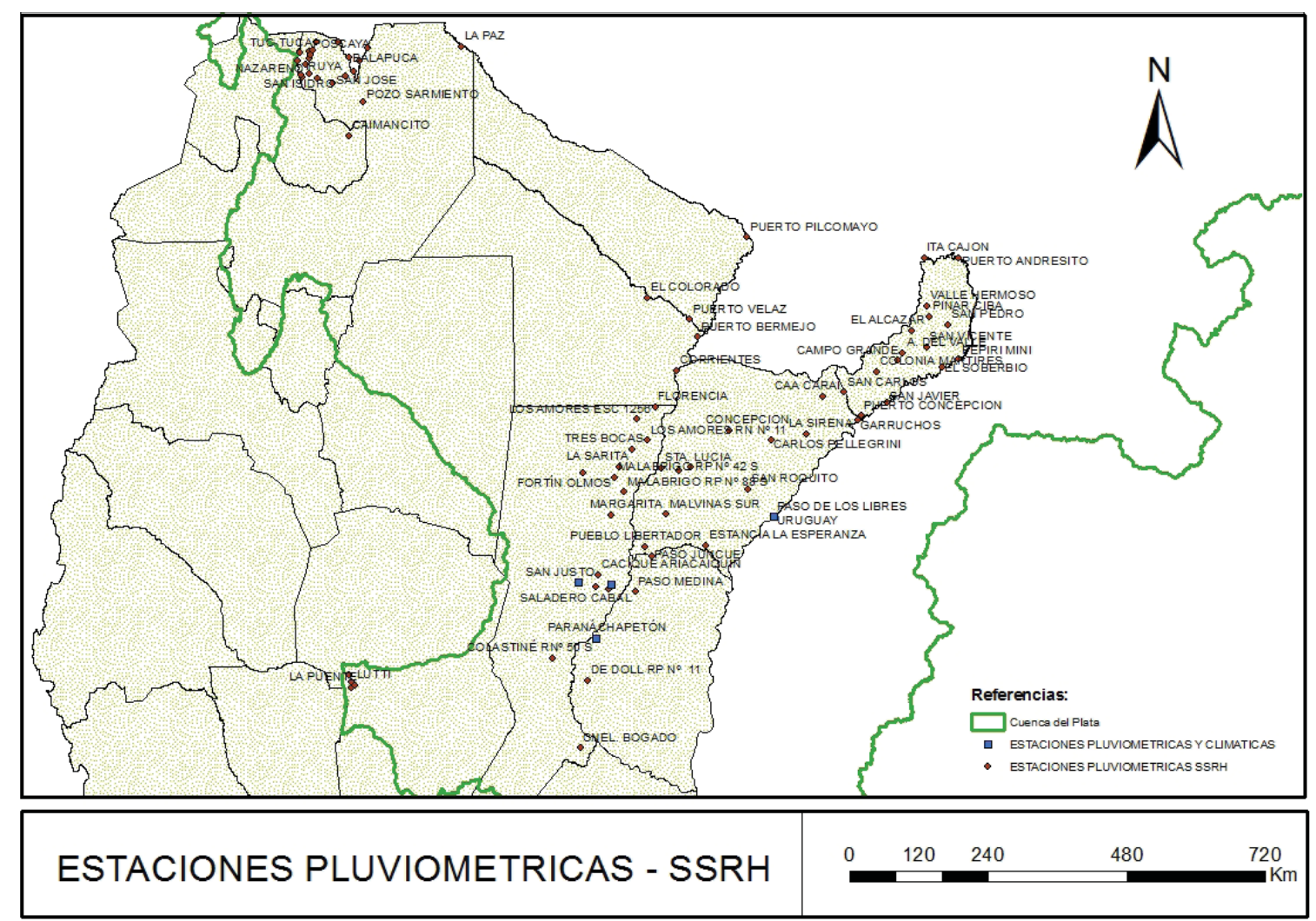

Fig. 2. Localización de las estaciones pluviométricas de la SSRH. 


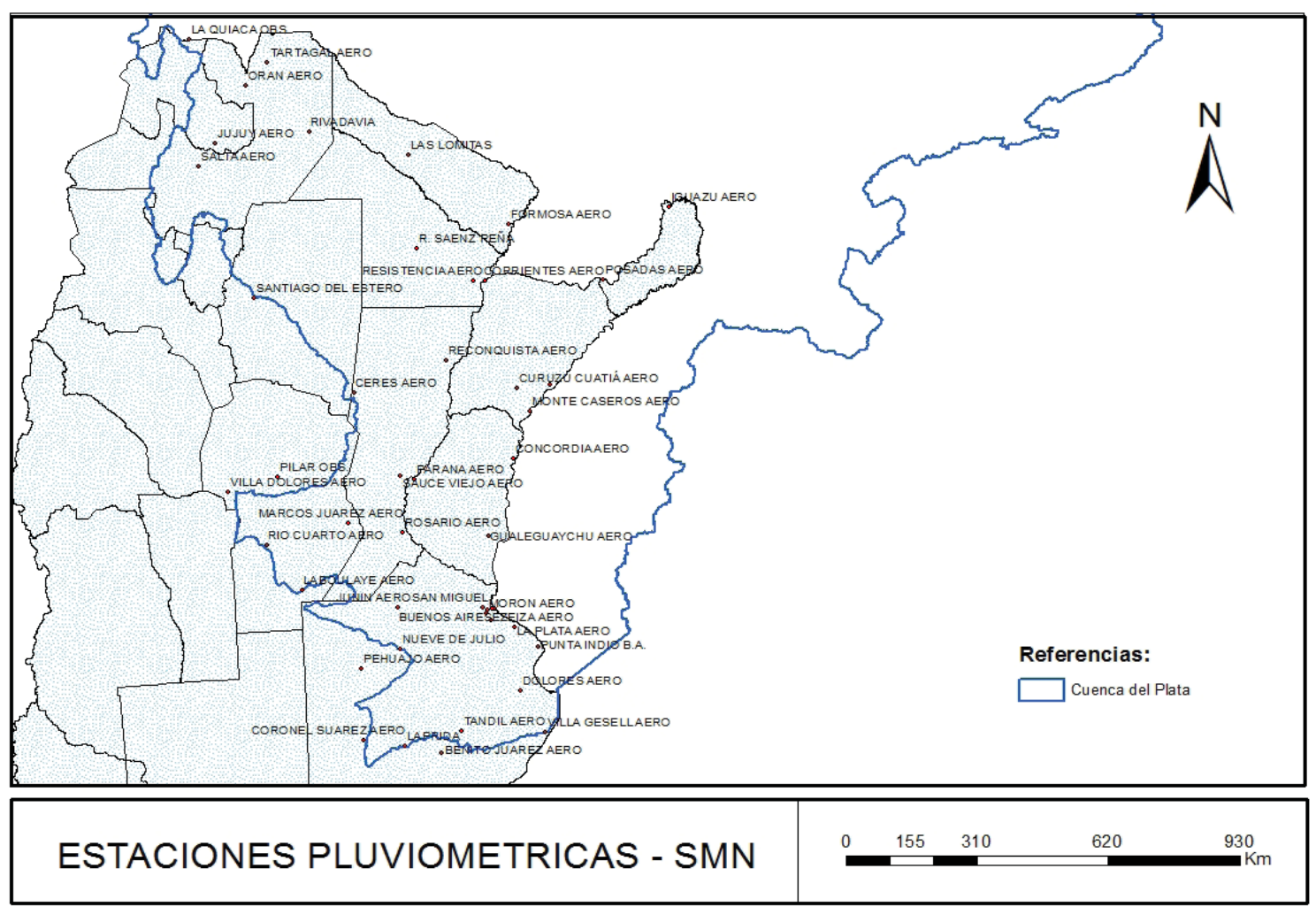

Fig. 3. Localización de las estaciones del SMN.

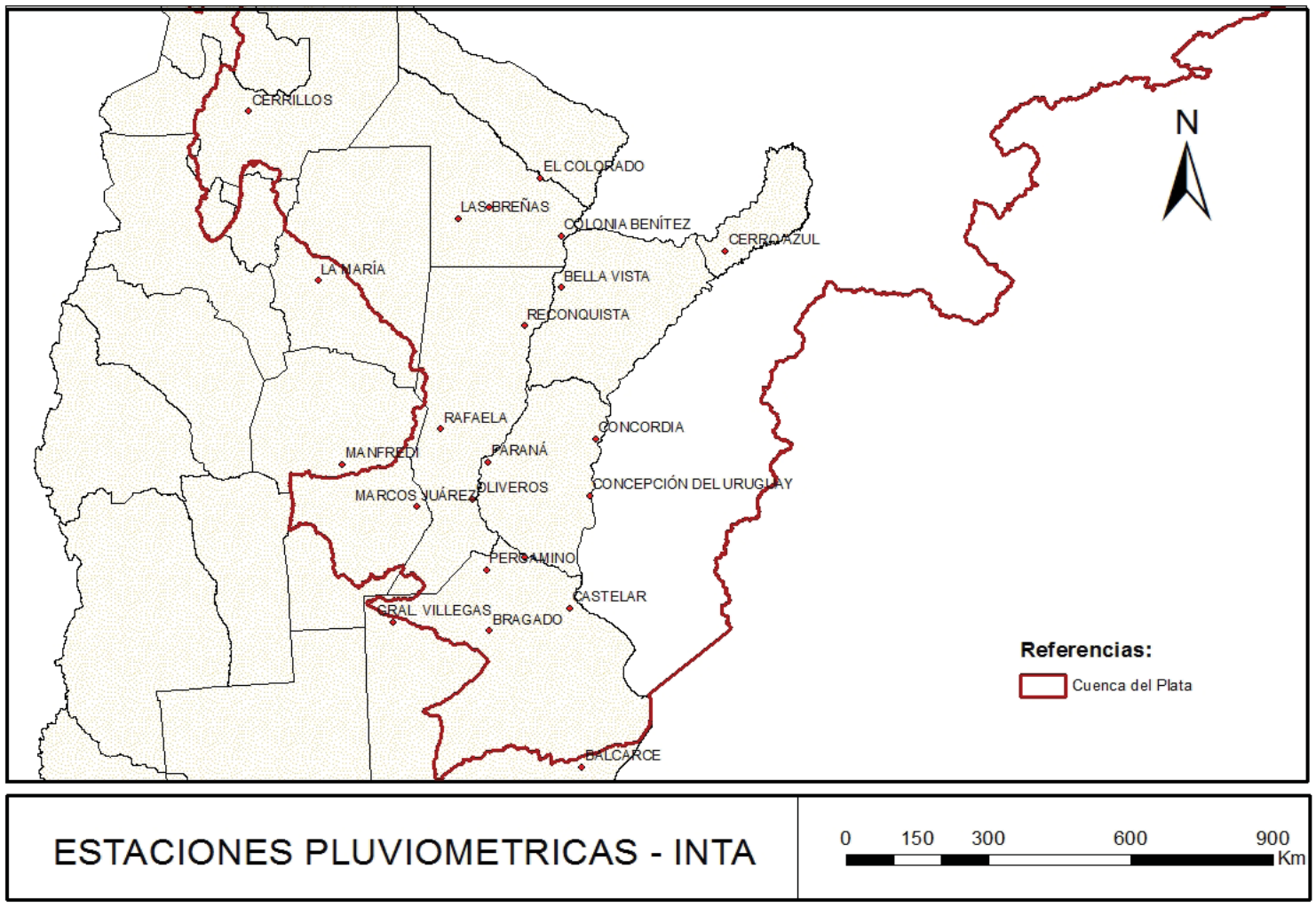

Fig. 4. Localización de las estaciones del INTA. 


\section{Lagunas de información y relleno de datos faltantes}

En primer lugar se determinaron la cantidad de datos mensuales faltantes, y como regla general no se usaron estaciones con menos de 25 años completos. Se realizaron los diagramas de disponibilidad de datos para la serie 1970-2010. A modo de ejemplo la Figura 5 muestra los registros de precipitación mensual para algunas estaciones pertenecientes a la SSRH en el década del 70. En estos diagramas se aprecian las lagunas de información y sirven para seleccionar períodos comunes de información entre estaciones.

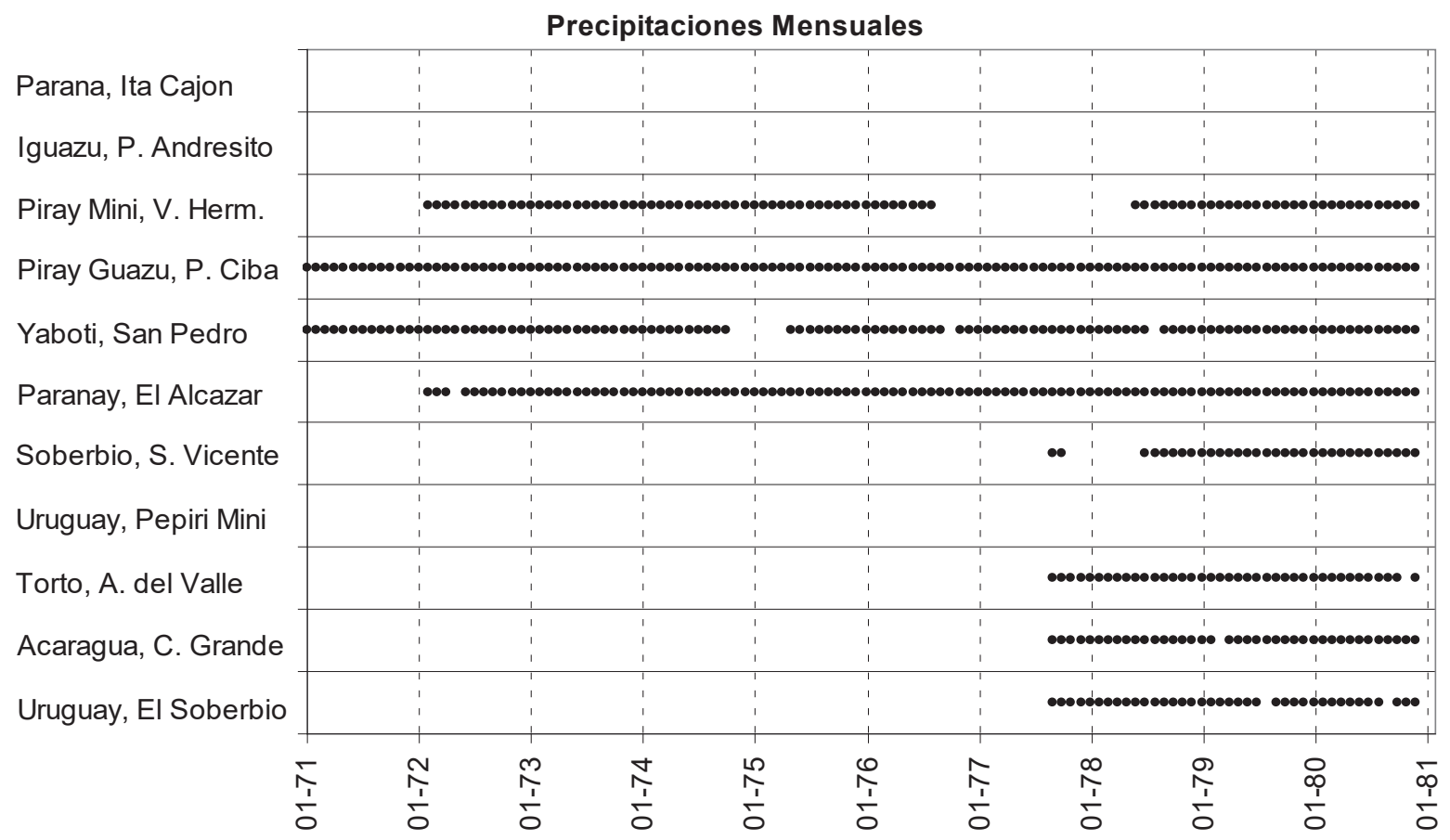

Fig. 5. Precipitaciones mensuales existentes SSRH. Período Ene 1971 a Dic 1980.

Se usaron procedimientos estadísticos apropiados para verificar la consistencia de los datos y para rellenar series con datos faltantes. Para completar las lagunas en las series de datos, se utiliza un modelo de regresión bivariado con estacionalización previa mensual de las series de datos, denominado CORMUL (CEDEX, 2003b). Este tratamiento se realizó mediante el Software CHAC desarrollado por el Centro de Estudios y Experimentación de Obras Publicas de España.

\section{Estimación de la ETP}

La estimación de la evapotranspiración potencial (ETP) se llevó a cabo mediante el método de Penman-Montheith, requiriéndose datos de temperatura, humedad relativa, velocidad de viento y heliofanía, los que no son comunes en todas la estaciones y una sola de estas variables que no se disponga ya limita su aplicación. Por este motivo quedaron afuera muchas estaciones que no conta- 
ban con las cuatro series de datos, disminuyendo la cantidad de estaciones posibles de utilizar. El cálculo de la ETP según Penman - Montheith se realizó directamente con el programa CHAC (CEDEX, 2003a).

\section{RESULTADOS Y DISCUSIÓN}

Distribución areal y temporal de las precipitaciones anuales

En la Tabla 1 se presenta el listado definitivo de las estaciones empleadas, para las series de 1970-71 al 2009-10, previamente rellenadas. Las mismas fueron conformadas en años hidrológicos de setiembre a agosto.

Fueron descartadas estaciones que no tuvieran suficientes registros y aquellas que no cumplieron los test de consistencia y homogeneidad, como dobles masas, test de Smirnov y test de Mann-Kendall.

Se aclara que si bien se requería que tuvieran al menos 25 años de datos completos, se aceptaron algunas estaciones que no tenían faltantes todo el año, sino algunos meses en varios años, con la finalidad de cubrir espacialmente la zona, tal es el caso de la estación Bragado que se mantuvo. 
Tabla 1a. Estaciones de precipitación utilizadas en la modelación.

\begin{tabular}{|c|c|c|c|c|c|c|c|}
\hline Estación & $\begin{array}{c}\text { Años } \\
\text { Completos }\end{array}$ & $\begin{array}{c}\text { P Media } \\
\text { anual }(\mathrm{mm})\end{array}$ & $\mathrm{CV}$ & CS & $\mathbf{C K}$ & Máx. & Mín. \\
\hline Iguazú Aero & 39 & 1883 & 0.24 & 0.79 & 0.71 & 2983 & 1091 \\
\hline Posadas Aero & 39 & 1802 & 0.23 & 1.20 & 1.83 & 3040 & 1212 \\
\hline Cerro Azul Inta & 40 & 1982 & 0.22 & 0.86 & 0.85 & 3146 & 1185 \\
\hline Corrientes Aero & 40 & 1468 & 0.24 & 0.59 & -0.11 & 2282 & 972 \\
\hline Colonia Benítez Inta & 40 & 1362 & 0.23 & 0.30 & -0.84 & 2010 & 828 \\
\hline R S Peña Inta & 40 & 1011 & 0.22 & 0.22 & -0.29 & 1484 & 588 \\
\hline Las Breñas Inta & 39 & 1001 & 0.22 & 0.59 & -0.05 & 1579 & 659 \\
\hline Resistencia Aero & 40 & 1412 & 0.22 & 0.54 & -0.42 & 2081 & 933 \\
\hline Bella Vista Inta & 40 & 1326 & 0.24 & 0.73 & 0.65 & 2262 & 751 \\
\hline Reconquista Inta & 39 & 1260 & 0.30 & 1.09 & 1.90 & 2487 & 693 \\
\hline P Libres Aero & 40 & 1561 & 0.27 & 1.13 & 2.38 & 3006 & 861 \\
\hline Curuzú Cuatiá Aero & 36 & 1375 & 0.27 & 1.14 & 3.01 & 2525 & 553 \\
\hline Monte Caseros Aero & 40 & 1494 & 0.24 & 0.61 & -0.64 & 2370 & 974 \\
\hline Ceres Aero & 40 & 966 & 0.26 & 0.22 & -0.43 & 1522 & 477 \\
\hline Rafaela Inta & 40 & 1024 & 0.26 & 0.66 & 0.25 & 1710 & 639 \\
\hline Sauce Viejo Aero & 39 & 1036 & 0.25 & 1.07 & 1.09 & 1779 & 690 \\
\hline Paraná Inta & 40 & 1110 & 0.25 & 0.68 & 0.52 & 1879 & 689 \\
\hline Concordia Inta & 35 & 1321 & 0.27 & 0.95 & 0.98 & 2295 & 740 \\
\hline C del Uruguay Inta & 40 & 1168 & 0.23 & 0.82 & 0.07 & 1841 & 736 \\
\hline Gualeguaychú Aero & 40 & 1143 & 0.24 & 0.78 & 0.08 & 1841 & 785 \\
\hline Oliveros Inta & 40 & 1031 & 0.21 & 0.30 & -1.05 & 1428 & 713 \\
\hline Rosario Aero & 40 & 1031 & 0.22 & 0.46 & -0.55 & 1482 & 635 \\
\hline Marcos Juárez Inta & 40 & 903 & 0.23 & 0.42 & -0.14 & 1426 & 524 \\
\hline Laboulaye Aero & 39 & 898 & 0.21 & 1.47 & 4.20 & 1608 & 595 \\
\hline San Pedro Inta & 39 & 1058 & 0.20 & -0.23 & -0.32 & 1437 & 553 \\
\hline Pergamino Inta & 39 & 1022 & 0.23 & 0.31 & -0.71 & 1507 & 629 \\
\hline Junín Aero & 39 & 1037 & 0.20 & 0.49 & 0.45 & 1577 & 653 \\
\hline Gral Villegas Inta & 36 & 859 & 0.21 & -0.98 & 2.71 & 1261 & 229 \\
\hline Bragado Inta & 13 & 1052 & 0.21 & 0.15 & -0.21 & 1552 & 535 \\
\hline Nueve de Julio Aero & 40 & 1068 & 0.20 & 0.00 & 0.12 & 1467 & 531 \\
\hline Pehuajó Aero & 40 & 967 & 0.21 & 0.63 & 1.07 & 1544 & 520 \\
\hline
\end{tabular}

(Leyenda: "P Media" corresponde a la precipitación media anual en $\mathrm{mm}$, de la serie con registros, "CV" es el coeficiente de variación adimensional, “CS” es el coeficiente de asimetría y “CK” es el coeficiente de curtosis) 
Tabla 1b. Estaciones de precipitación utilizadas en la modelación.

\begin{tabular}{|c|c|c|c|c|c|c|c|}
\hline Estación & $\begin{array}{c}\text { Años } \\
\text { Completos }\end{array}$ & $\begin{array}{c}\text { P Media } \\
\text { anual }(\mathbf{m m})\end{array}$ & $\mathrm{CV}$ & CS & CK & Máx. & Mín. \\
\hline San Miguel Aero & 40 & 1169 & 0.15 & -0.37 & 0.27 & 1579 & 779 \\
\hline Aeroparque Aero & 40 & 1077 & 0.18 & -0.25 & -0.43 & 1449 & 685 \\
\hline Castelar Inta & 40 & 1039 & 0.16 & 0.25 & 0.58 & 1477 & 674 \\
\hline Ezeiza Aero & 40 & 1005 & 0.19 & 0.37 & 0.11 & 1451 & 660 \\
\hline La Plata Aero & 38 & 1054 & 0.20 & 0.29 & 0.28 & 1652 & 622 \\
\hline Punta Indio Aero & 36 & 987 & 0.25 & -0.21 & 0.24 & 1478 & 342 \\
\hline Dolores Aero & 35 & 965 & 0.19 & -0.05 & 0.35 & 1434 & 553 \\
\hline Coronel Suárez Aero & 38 & 806 & 0.23 & 0.42 & 0.14 & 1250 & 424 \\
\hline Laprida Aero & 27 & 845 & 0.21 & 0.45 & 1.11 & 1348 & 411 \\
\hline Tandil Aero & 40 & 902 & 0.22 & 0.03 & 0.01 & 1347 & 459 \\
\hline Benito Juárez Aero & 24 & 883 & 0.17 & 0.86 & 2.53 & 1405 & 548 \\
\hline Balcarce Inta & 40 & 918 & 0.19 & 0.27 & -0.25 & 1347 & 596 \\
\hline Puerto Bermejo & 24 & 1256 & 0.30 & -0.16 & -0.45 & 1938 & 427 \\
\hline Paraná, Chapetón & 31 & 1105 & 0.26 & 0.67 & 0.01 & 1829 & 645 \\
\hline El Toba, Margarita & 27 & 1142 & 0.30 & 0.85 & 0.10 & 1990 & 666 \\
\hline Fortín Olmos & 32 & 1110 & 0.31 & 0.57 & -0.23 & 1879 & 532 \\
\hline Malabrigo, RP88 & 24 & 1249 & 0.29 & 1.19 & 2.46 & 2474 & 606 \\
\hline Saladero Cabal & 25 & 1124 & 0.27 & 0.34 & -0.15 & 1768 & 504 \\
\hline Los Amores, RN11 & 30 & 1348 & 0.24 & 0.57 & 0.15 & 2226 & 741 \\
\hline El Rey, La Sarita & 30 & 1240 & 0.28 & 0.74 & 0.57 & 2195 & 606 \\
\hline Colastiné, RP50 & 23 & 999 & 0.17 & -0.02 & -0.62 & 1369 & 614 \\
\hline Las Garzas, 3 Bocas & 25 & 1282 & 0.25 & 0.36 & 1.13 & 2217 & 563 \\
\hline Malabrigo, RP42 & 24 & 1232 & 0.26 & 0.77 & 1.15 & 2194 & 676 \\
\hline Pavón, Cnel Bogado & 21 & 933 & 0.21 & 0.29 & -0.65 & 1346 & 560 \\
\hline Cacique Araicaiquin & 25 & 1097 & 0.27 & 0.30 & -0.80 & 1764 & 668 \\
\hline San Justo & 27 & 1035 & 0.23 & 0.03 & -0.14 & 1568 & 528 \\
\hline Colonia Mascias & 23 & 1123 & 0.25 & 0.23 & -0.38 & 1689 & 617 \\
\hline Florencia & 26 & 1351 & 0.21 & 0.25 & 0.73 & 2047 & 721 \\
\hline Pinar Ciba & 31 & 1843 & 0.24 & 0.66 & 0.39 & 3046 & 1096 \\
\hline Colonia Mártires & 34 & 1958 & 0.23 & 1.48 & 2.46 & 3341 & 1243 \\
\hline Valle Hermoso & 30 & 1807 & 0.22 & 0.68 & 0.19 & 2907 & 1042 \\
\hline
\end{tabular}

(Leyenda: "P Media" corresponde a la precipitación media anual en $\mathrm{mm}$, de la serie con registros, " $C V$ ” es el coeficiente de variación adimensional, "CS” es el coeficiente de asimetría y " CK” es el coeficiente de curtosis) 
Tabla 1c. Estaciones de precipitación utilizadas en la modelación.

\begin{tabular}{|c|c|c|c|c|c|c|c|}
\hline Estación & $\begin{array}{c}\text { Años } \\
\text { Completos }\end{array}$ & $\begin{array}{c}\text { P Media } \\
\text { anual (mm) }\end{array}$ & $\mathrm{CV}$ & CS & $\mathbf{C K}$ & Máx. & Mín. \\
\hline Campo Grande & 24 & 1890 & 0.21 & 0.96 & 1.68 & 3105 & 1119 \\
\hline El Alcázar & 37 & 1894 & 0.23 & 0.40 & 0.16 & 2923 & 1010 \\
\hline San Pedro & 27 & 2077 & 0.18 & 0.54 & 1.71 & 3275 & 1343 \\
\hline Torto, A del Valle & 20 & 1959 & 0.20 & 0.42 & 0.48 & 3011 & 1188 \\
\hline San Vicente & 23 & 1913 & 0.19 & 0.47 & -0.12 & 2777 & 1196 \\
\hline El Soberbio & 24 & 1863 & 0.20 & 1.32 & 3.06 & 3058 & 1188 \\
\hline Pepiri Mini & 21 & 1781 & 0.20 & 0.65 & 1.17 & 2807 & 1148 \\
\hline Puerto Andresito & 25 & 1786 & 0.20 & 0.60 & 0.34 & 2597 & 1128 \\
\hline Ita Cajón & 22 & 1556 & 0.26 & 0.65 & 0.16 & 2553 & 845 \\
\hline Paso Juncué & 24 & 1274 & 0.23 & 0.56 & 0.74 & 2111 & 769 \\
\hline La Sirena & 25 & 1722 & 0.30 & 0.98 & 0.97 & 3096 & 891 \\
\hline Carlos Pellegrini & 29 & 1356 & 0.28 & 0.60 & 0.54 & 2437 & 727 \\
\hline San Roquito & 23 & 1270 & 0.27 & 0.30 & -0.32 & 2068 & 674 \\
\hline Santa Lucía & 32 & 1113 & 0.35 & 0.10 & -1.04 & 1759 & 463 \\
\hline Batel-Batelito, Concp & 32 & 1496 & 0.28 & 0.51 & 0.99 & 2628 & 594 \\
\hline Paso Cerrito & 26 & 1242 & 0.29 & 0.93 & 1.46 & 2304 & 660 \\
\hline Garruchos & 21 & 1686 & 0.25 & 1.08 & 2.44 & 3074 & 848 \\
\hline Caa Caraí & 21 & 1469 & 0.23 & 0.95 & 1.22 & 2546 & 929 \\
\hline San Carlos & 23 & 1735 & 0.20 & 0.75 & 0.73 & 2690 & 1122 \\
\hline Corrientes, Chavarría & 27 & 1285 & 0.28 & 0.71 & 0.30 & 2239 & 569 \\
\hline Pueblo Libertador & 25 & 1238 & 0.27 & 0.84 & 1.59 & 2286 & 649 \\
\hline Estcia. La Esperanza & 30 & 1296 & 0.27 & 1.23 & 1.76 & 2449 & 846 \\
\hline Malvinas Sur & 33 & 1147 & 0.31 & 0.78 & 0.10 & 2125 & 605 \\
\hline Rivadavia & 26 & 642 & 0.25 & -0.42 & 0.42 & 1009 & 329 \\
\hline Salta Aero & 39 & 744 & 0.20 & -0.04 & 0.76 & 1018 & 428 \\
\hline Las Lomitas & 40 & 933.7 & 0.18 & 0.86 & 1.62 & 1422 & 604 \\
\hline Río Cuarto Aero & 39 & 856 & 0.19 & 0.22 & -0.45 & 1231 & 574 \\
\hline Stgo del Estero & 39 & 499 & 0.54 & 0.36 & 0.79 & 1284 & 80 \\
\hline Campo Gallo & 39 & 691 & 0.27 & 0.78 & 1.93 & 1281 & 329 \\
\hline Caimancito & 35 & 1063 & 0.24 & 0.51 & -0.12 & 1035 & 660 \\
\hline Potrero del Clavillo & 40 & 1059 & 0.19 & 0.17 & 0.17 & 1041 & 609 \\
\hline El Colorado & 39 & 1167 & 0.25 & 1.00 & 1.51 & 2118 & 768 \\
\hline
\end{tabular}

(Leyenda: "P Media” corresponde a la precipitación media anual en $\mathrm{mm}$, de la serie con registros, "CV” es el coeficiente de variación adimensional, “CS” es el coeficiente de asimetría y "CK” es el coeficiente de curtosis) 
Se destacan altas asimetrías positivas para la precipitación media anual en las estaciones de Colonia Mártires $(\mathrm{CS}=1.48)$ y Laboulaye $(\mathrm{CS}=1.47)$, acompañadas de coeficiente de curtosis elevados. El mayor coeficiente de variabilidad corresponde a Santiago del Estero con un valor de 0.54. E1 rango del coeficiente de variabilidad esta entre 0.15 y 0.54 , mientras que el coeficiente de asimetría varía entre -0.98 en Gral Villegas y 1.48 .

A las series de precipitaciones anuales, se le realizaron las gráficas de dobles acumuladas a los efectos de detectar errores presentes en los datos, en la mayoría de los casos se mantiene una buena proporcionalidad entre las estaciones contrastadas, excepto para el caso de algunas estaciones de la prov. de Buenos Aires y de la prov. de Corrientes, además de la estación Iguazú Aero en Misiones.

Luego se analiza en cada estación la precipitación media cronológica incremental, la cual es de utilidad para el análisis de tendencias. Se adjuntan la representación correspondiente a la estación Resistencia Aero en Chaco (Figura 6), en ella se muestra para el período 1971-2010, el promedio de la serie, los valores anuales y las medias cronológicas incrementales.

Resistencia Aero

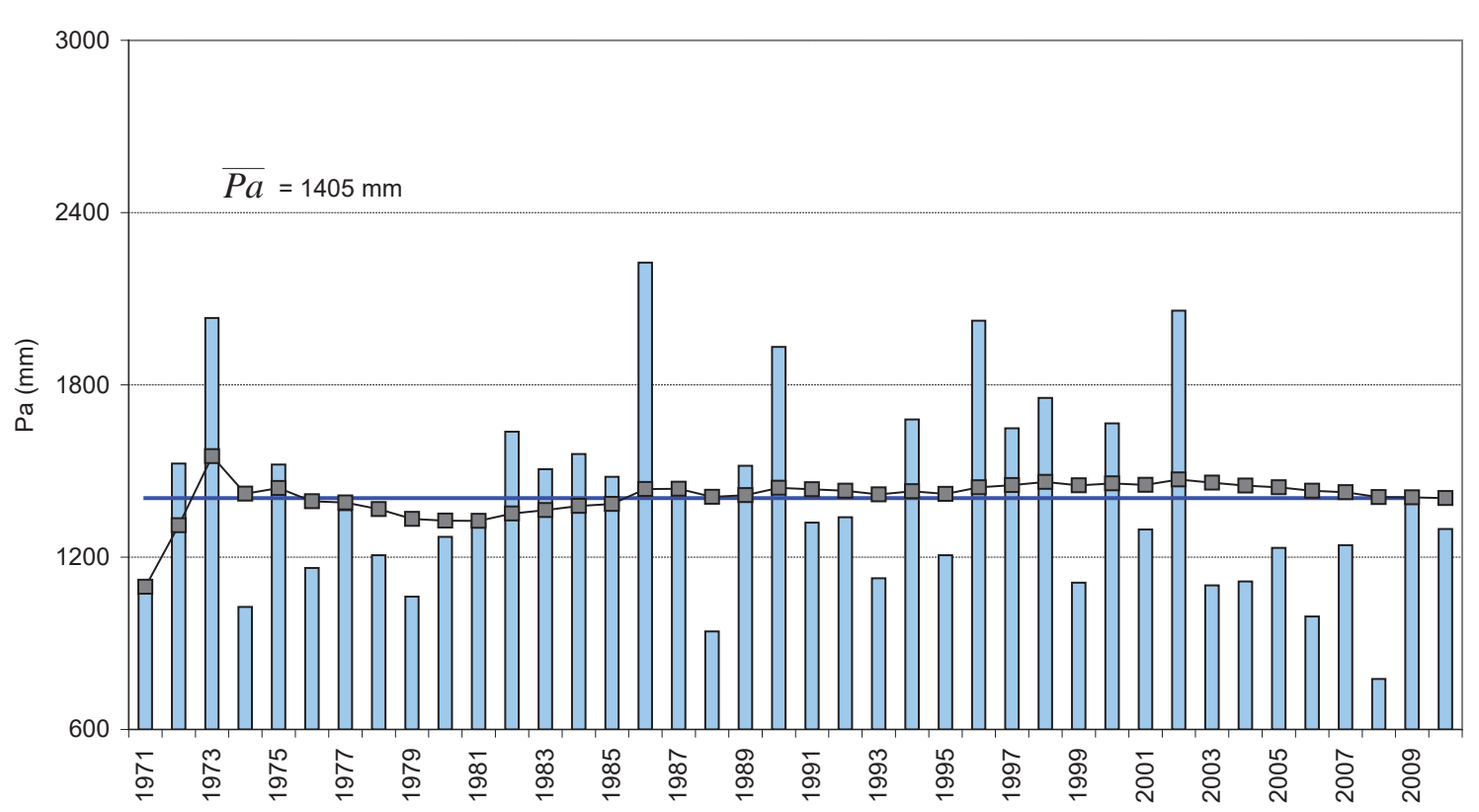

Fig. 6. Precipitación anual y medias cronológicas incrementales. Resistencia Aero. Serie 1971-2010.

A partir de las series y figuras analizadas se observa la característica general que presenta el régimen de precipitaciones anuales en la región que es la tendencia a agrupar años húmedos (por encima de la media), años secos (por debajo de la media) en períodos de entre 3 a 5 años e intercalarse con otros períodos de alternancia (por encima y por debajo de la media).

Claramente estos agrupamientos no son homogéneos para toda la región, pero si se encuentra correspondencia entre las estaciones más próximas. 
En cuanto a la posible tendencia de aumento o disminución de los montos anuales de lluvia, en general se presenta en la mayor parte de la región una tendencia al aumento de precipitación anual desde los años $70 \mathrm{u}$ 80 hasta el 2000 y luego una leve tendencia a la disminución hacia el año 2010. Conviene resaltar que el análisis es exclusivamente referido al período 1970 - 2010 y no hace ninguna comparación con períodos anteriores.

La tendencia general expresada se manifiesta con variada intensidad según las subregiones que se consideren.

En el extremo noreste (prov. de Misiones), se denota el crecimiento leve desde los años 80 hasta el 2000, a partir del cual se mantiene estable, mientras que las zonas este del Chaco, norte de Santa Fe y en Corrientes el decrecimiento es más marcado a partir del año 2002.

En la parte centro y sur de la prov. de Santa Fe y sur de la prov. de Entre Ríos, no se manifiesta esta declinación en los últimos 10 años, justamente porque se presenta un agrupamiento manifiesto de años húmedos entre el 2000 y 2003 y el año 2007.

En la zona centro-oeste de la región se manifiesta una leve tendencia a la disminución de la precipitación anual de los 80 a la actualidad.

A partir de esta información se ha realizado el trazado de isohietas anuales en $\mathrm{mm}$, para el período 1970-2010 que se presenta en la Figura 7.

En términos generales la variación de la precipitación anual va disminuyendo desde las zonas más húmedas (este) hacia las más áridas (oeste). Sin embargo, la presencia de la precordillera de los Andes, hace de barrera orográfica, logrando que las nubes descarguen su humedad, aumentando los montos de precipitación en la franja que se encuentra sobre las provincias de Salta y Jujuy. En esta zona si bien los montos de precipitación anual son elevados, la distribución en el año de la precipitación se concentra en los meses de verano, resultando los inviernos fríos y secos con una marcada estacionalidad.

En conclusión el gradiente precipitación va aumentando de este a oeste, siendo en la mitad superior de la cuenca del orden de los $2200 \mathrm{~mm}$ en Misiones, hasta $700 \mathrm{~mm}$ en el este de Formosa y el Chaco, así como en gran parte de Santiago del Estero; e incluso hacia el sur, valores inferiores a $600 \mathrm{~mm}$ anuales. Luego, más hacia al noroeste, debido a la barrera orográfica de la precordillera de los Andes, vuelven a aumentar hasta máximos de $1600 \mathrm{~mm}$. En tanto, para la mitad inferior de la cuenca las precipitaciones anuales varían entre 1400 en el sur de Corrientes, hasta $800 \mathrm{~mm}$ en algunas zonas del oeste de Santa Fe, e incluso $700 \mathrm{~mm}$.

En relación a la variabilidad espacial, la menor se localiza en las zonas de la Mesopotamia y el Chaco Húmedo, donde la cercanía con las grandes masas de agua que son los ríos Paraná y Paraguay, atenúan el efecto de variabilidad climática, incluso en la zona sur de la cuenca la variación es suave. A su vez, el mayor gradiente se presenta en las provincias de Salta y Jujuy, con una brusca variación, que se manifiesta en las isohietas más apretadas. 

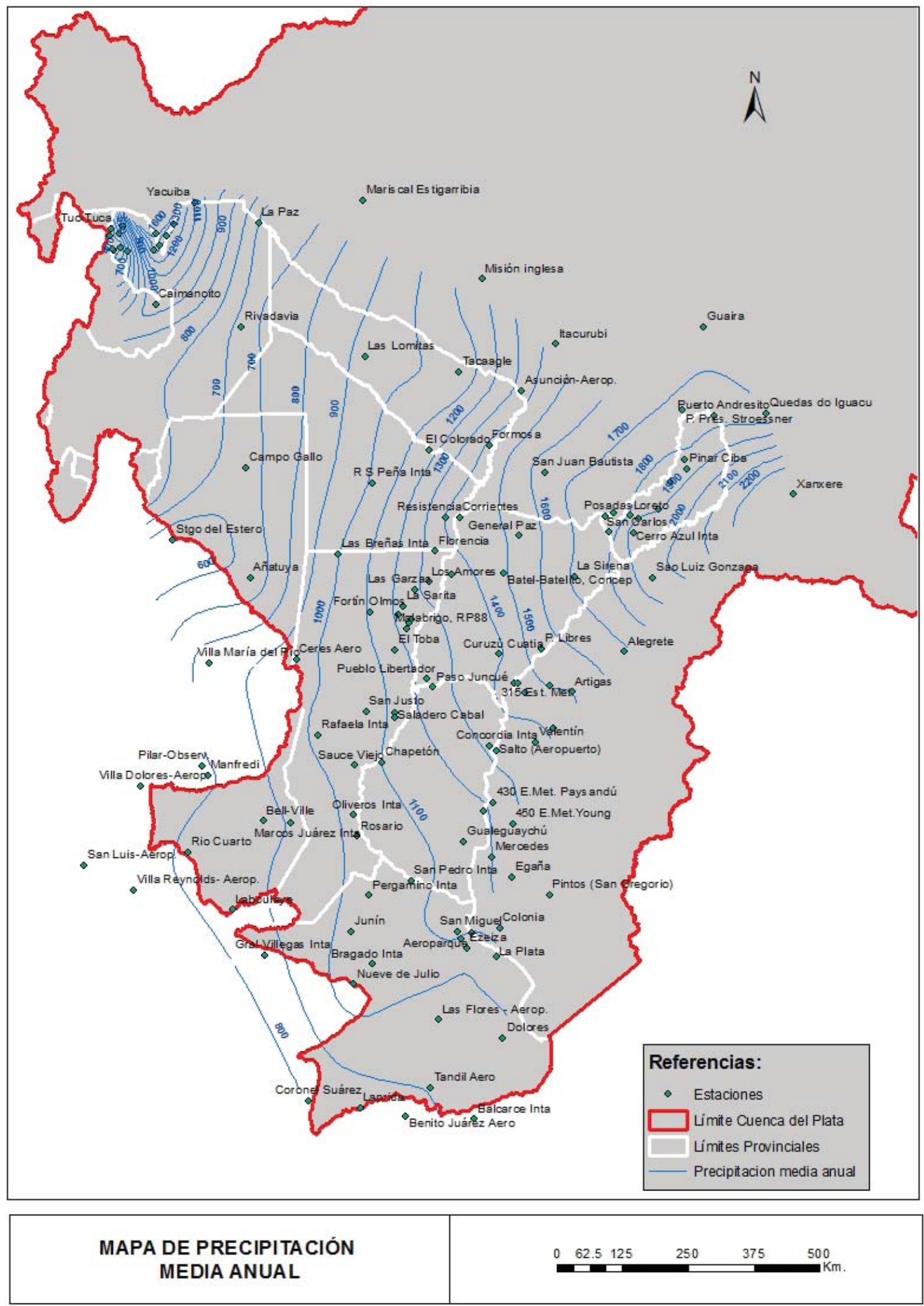

Fig. 7. Isohietas medias anuales zona de estudio. Período 1970-2010. 


\section{Distribución anual de precipitaciones mensu- ales}

Se confeccionaron planillas con los datos de precipitación mensuales de cada estación para la serie 1970-2010 y sus correspondientes valores promedios, máximos y mínimos, de las series mensuales y anuales. Como ejemplo se incluye en el Anexo I la Tabla de la estación Resistencia Aero.
A partir de los valores medios mensuales de cada estación del período 1970-2010, se han trazado las isohietas de cada mes para cada estación. Se analiza a partir de considerar los valores de precipitación promedio de cada mes para toda la serie disponible, 1970 - 2010, y su graficación. A modo de ejemplo se adjunta la figura correspondiente a la estación Resistencia Aero en Chaco (Figura 8).

\section{Precipitación media mensual - Resistencia Aero}

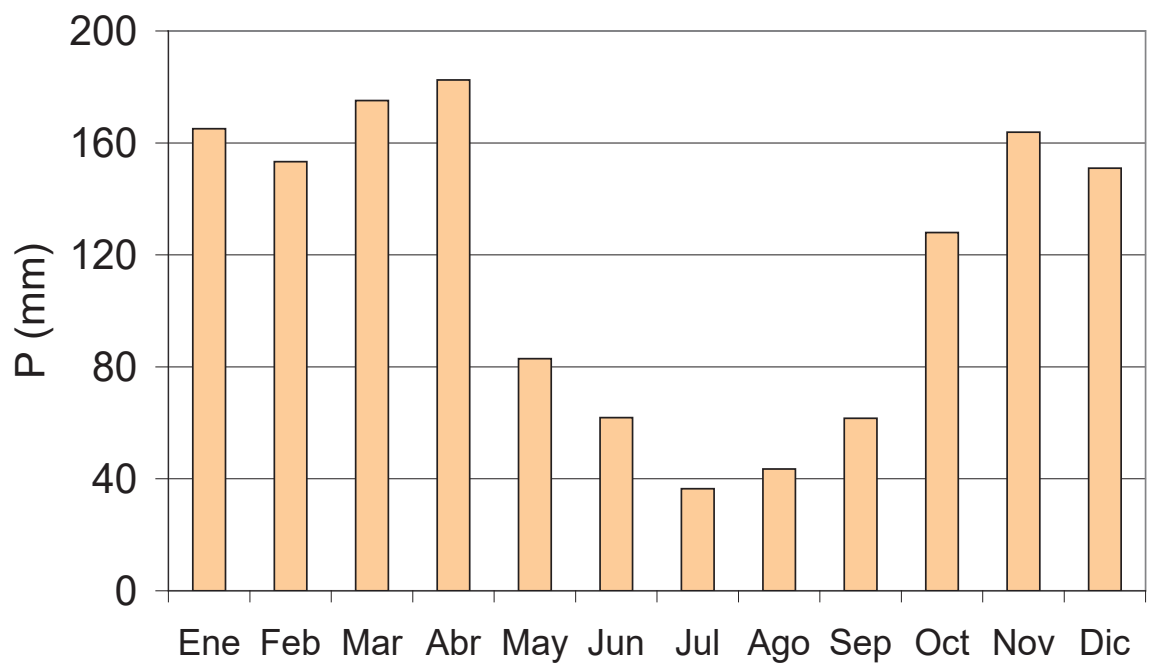

Fig. 8. Precipitación media mensual. Resistencia Aero. Serie 1970-2000.

La tendencia general en toda la región indica un ciclo unimodal, con mínimos en invierno y máximos en verano-otoño, no homogéneo sobre todo desde el punto de vista de la disminución invernal de las lluvias. En el extremo nordeste de la región esta disminución invernal es poco marcada y se va acentuando hacia el oeste, siendo muy marcada en las estaciones de Córdoba y Santiago del Estero.

Sin embargo esta distribución a lo largo del año en valores medios, no tiene más valor que una síntesis estadística, puesto que a nivel de años reales, la variabilidad de las precipitaciones mensuales es muy grande con valores que pueden ser 10 veces más grandes o más chicos que el valor medio, por lo tanto los datos no pueden ser interpretados de un modo predictivo.

Asimismo es frecuente observar el fenómeno de persistencia por el cual se dan sucesión de 2, 3 o más meses seguidos de situaciones tanto de excesos como de déficit.

Para completar la información, en el Anexo II se presentan las medias mensuales y anuales de cada estación para la serie 1970/71-2009/10.

\section{Análisis de la información meteorológica usa- da para evaluar la ETP}

Del mismo modo que se procedió con la información de precipitación, se analizaron 
los datos mensuales faltantes de las variables temperatura, humedad relativa, velocidad de viento y heliofonía para retener únicamente las estaciones que sirvan a la determinación de la evapotranspiración areal de las cuencas. En este sentido se procedió a descartar aquellas estaciones que tenían muchos años faltantes, que se superponían localmente o que no disponían de alguna de las cuatro variables que requiere el método de Penman-Monteith. Resultando entonces, un total de 38 estaciones posibles de utilizar. Las mismas, con sus valores medios, se presentan en la Tabla 2.

Tabla 2. Estaciones con datos meteorológicos utilizadas.

\begin{tabular}{|c|c|c|c|c|c|c|c|c|}
\hline \multirow[b]{2}{*}{ Estación } & \multicolumn{2}{|c|}{ Temperatura } & \multicolumn{2}{|c|}{ Humedad Relat } & \multicolumn{2}{|c|}{ Heliofanía } & \multicolumn{2}{|c|}{ Veloc. Viento } \\
\hline & $\begin{array}{c}\text { Años } \\
\text { Comple- } \\
\text { tos }\end{array}$ & $\begin{array}{c}\text { Media } \\
\text { anual } \\
\left({ }^{\circ} \mathrm{C}\right)\end{array}$ & $\begin{array}{c}\text { Años } \\
\text { Comple- } \\
\text { tos }\end{array}$ & $\begin{array}{c}\text { Media } \\
\text { anual } \\
(\%)\end{array}$ & $\begin{array}{c}\text { Años } \\
\text { Comple- } \\
\text { tos }\end{array}$ & $\begin{array}{c}\text { Media } \\
\text { anual } \\
\text { (hs) }\end{array}$ & $\begin{array}{c}\text { Años } \\
\text { Comple- } \\
\text { tos }\end{array}$ & $\begin{array}{c}\text { Media } \\
\text { anual } \\
(\mathrm{km} / \mathrm{d})\end{array}$ \\
\hline Iguazú Aero & 33 & 21 & 34 & 80.4 & 18 & 1885.3 & 34 & 163.5 \\
\hline Posadas Aero & 39 & 21.6 & 39 & 72.6 & 26 & 2123.9 & 39 & 290.1 \\
\hline Cerro Azul Inta & 40 & 21.2 & 40 & 71.1 & 40 & 2529.9 & 40 & 162.4 \\
\hline Corrientes Aero & 40 & 21.2 & 40 & 74.6 & 30 & 2690.2 & 40 & 314.3 \\
\hline $\begin{array}{l}\text { Colonia Benitez } \\
\text { Inta }\end{array}$ & 40 & 21.5 & 40 & 74.2 & 40 & 2620.9 & 40 & 233.6 \\
\hline R S Peña Inta & 39 & 21.5 & 40 & 70.1 & 40 & 2656.2 & 40 & 214.4 \\
\hline Las Breñas Inta & 39 & 28 & 39 & 65 & 38 & 2667.6 & 39 & 274.5 \\
\hline Resistencia A & 39 & 21 & 39 & 75.4 & 27 & 2204.7 & 39 & 224 \\
\hline Bella Vista Inta & 39 & 21.1 & 37 & 72.5 & 35 & 2499.4 & 39 & 215.3 \\
\hline Reconquista Inta & 39 & 20.1 & 37 & 75.5 & 24 & 2570.3 & 39 & 219 \\
\hline P Libres Aero & 39 & 19.8 & 39 & 74 & 27 & 2123.8 & 38 & 348.7 \\
\hline $\begin{array}{l}\text { Montecase-ros } \\
\text { Aero }\end{array}$ & 40 & 19.6 & 40 & 74.3 & 33 & 2628.6 & 40 & 224.7 \\
\hline Ceres Aero & 40 & 18.9 & 40 & 75.4 & 30 & 2647.9 & 39 & 275.5 \\
\hline Rafaela Inta & 40 & 18.4 & 35 & 72.6 & 40 & 2679.9 & 34 & 282.7 \\
\hline Sauce Viejo Aero & 39 & 18.7 & 39 & 76.5 & 22 & 2662.6 & 39 & 300.6 \\
\hline Paraná Inta & 40 & 18.6 & 40 & 70.7 & 37 & 2618.9 & 40 & 311.4 \\
\hline Concordia Inta & 30 & 18.8 & 28 & 75.4 & 19 & 2557.2 & 24 & 161 \\
\hline $\begin{array}{l}\text { C del Uruguay } \\
\text { Inta }\end{array}$ & 40 & 18.1 & 38 & 70.4 & 39 & 2596.9 & 38 & 234.3 \\
\hline $\begin{array}{l}\text { Gualeguay-chú } \\
\text { Aero }\end{array}$ & 40 & 17.8 & 40 & 73.9 & 31 & 2315.9 & 40 & 220.1 \\
\hline Oliveros Inta & 40 & 18 & 40 & 74 & 40 & 2609 & 40 & 268.9 \\
\hline Rosario Aero & 40 & 17.4 & 40 & 74.7 & 30 & 2632.8 & 39 & 348.7 \\
\hline $\begin{array}{l}\text { Marcos Juárez } \\
\text { Inta }\end{array}$ & 40 & 17.7 & 40 & 70 & 34 & 2600 & 40 & 216.8 \\
\hline Laboulaye Aero & 40 & 16 & 39 & 71.8 & 33 & 2648.1 & 40 & 322.5 \\
\hline
\end{tabular}




\begin{tabular}{|l|c|c|c|c|c|c|c|c|}
\hline San Pedro Inta & 40 & 17.2 & 40 & 73.1 & 38 & 2556.1 & 40 & 229.5 \\
\hline Pergamino Inta & 40 & 16.7 & 40 & 70.8 & 40 & 2638 & 38 & 269.8 \\
\hline Junín Aero & 40 & 15.9 & 40 & 73.9 & 34 & 2538.6 & 40 & 291.6 \\
\hline Gral Villegas Inta & 19 & 15.9 & 11 & 70.4 & 13 & 2605.8 & 9 & 331.5 \\
\hline Pehuajo Aero & 39 & 15.4 & 39 & 75.1 & 31 & 2611.8 & 39 & 286.1 \\
\hline San Miguel Aero & 37 & 16.9 & 37 & 75.8 & 30 & 2597.9 & 37 & 212.1 \\
\hline Aeroparque Aero & 40 & 17.8 & 40 & 72.5 & 31 & 2478.8 & 40 & 381.7 \\
\hline Castelar Inta & 40 & 17 & 40 & 69.2 & 40 & 2633 & 40 & 198 \\
\hline Ezeiza Aero & 39 & 16.6 & 39 & 73.6 & 23 & 1911.1 & 39 & 319.1 \\
\hline La Plata Aero & 37 & 15.9 & 37 & 78.8 & 27 & 2541.5 & 36 & 363.6 \\
\hline Punta Indio Aero & 34 & 16 & 34 & 81.5 & 30 & 2449.1 & 34 & 368.1 \\
\hline Dolores Aero & 30 & 14.9 & 30 & 79.8 & 27 & 1986.1 & 30 & 253.9 \\
\hline Cnel Suárez Aero & 31 & 13.5 & 31 & 73.9 & 30 & 2531.3 & 31 & 276.5 \\
\hline Tandil Aero & 39 & 13.6 & 39 & 75.4 & 24 & 2297.5 & 40 & 346.4 \\
\hline Balcarce Inta & 40 & 14 & 40 & 77.7 & 40 & 2327.5 & 35 & 260 \\
\hline
\end{tabular}

Para rellenar los datos mensuales de las variables requeridas por el método se utilizó el mismo procedimiento empleado con la información de precipitación, Se destaca que la mayoría de las estaciones tenía las series casi completas, siendo la heliofanía la variable con más lagunas.

\section{Distribución areal y temporal de la ETP anual}

Los valores medios mensuales de la evapotranspiración potencial para la serie 1970/71-2009/10 en cada estación se muestran en el Anexo III.

Se mencionó anteriormente la dificultad para lograr estaciones que dispusieran de las cuatro variables necesarias para estimarla, no obstante esta complejidad para su determinación, la variabilidad que presenta en forma espacial, en el territorio argentino de cuenca del Plata, no es tan marcada, lo que de alguna manera justifica la menor cantidad de puntos de representación.

La Figura 9 presenta las isolíneas de evapotranspiración potencial anuales en el periodo 1970-2010 en la zona de estudio. En ella se observa un gradiente en aumento desde el sureste al noroeste con valores que van desde los $1000 \mathrm{~mm}$, en la provincia de Buenos Aires, hasta $1700 \mathrm{~mm}$ en Santiago del Estero y Salta. Sin embargo en las zonas con relieve más elevado en el oeste de Salta y en Jujuy la ETP tiende a disminuir, hasta cifras del orden de $1300 \mathrm{~mm}$.

Aunque los máximos de precipitación y los mínimos de ETP no se superponen totalmente, en términos generales, en las zonas de llanura, las precipitaciones más elevadas y las evapotranspiraciones más bajas corresponden al este de la Cuenca del Plata, en donde a escala anual se aprecia que $\mathrm{P}>$ ETP. $\mathrm{La}$ aridez presenta tendencia a incrementarse hacia el oeste, de modo que buena parte de Formosa, Chaco, Santiago del Estero y la zona más oriental de Salta presentan importantes déficits hídricos.

$\mathrm{Si}$ se analiza temporalmente, utilizando las series de ETP anuales de todas las estaciones, se encuentra que los valores extremos 


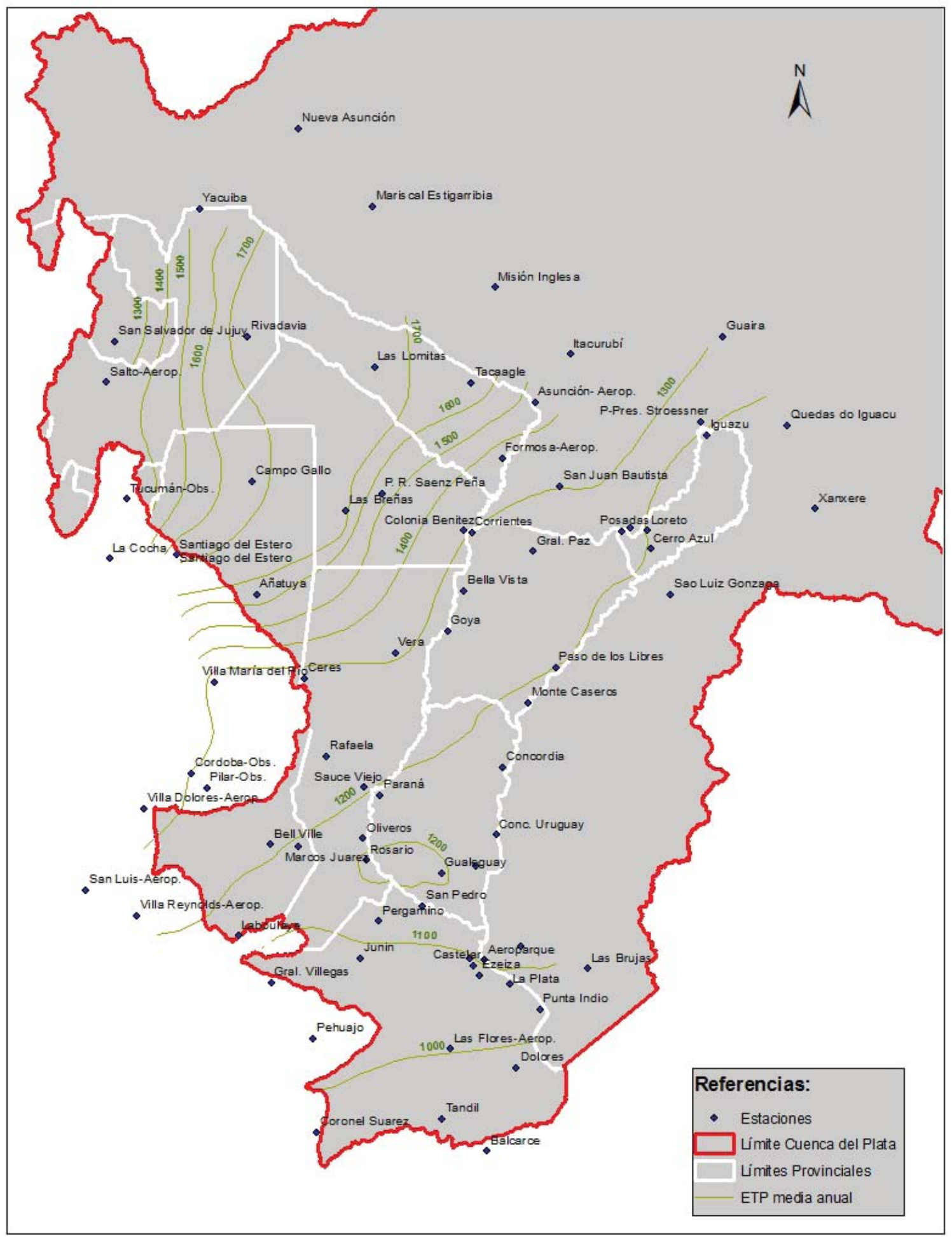

MAPA DE EVAPOTRAN SPIRACIÓN POTENCIAL MEDIA ANUAL

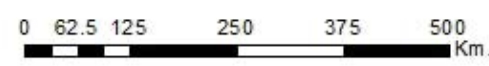

Fig. 9. Isolíneas de ETP medias anuales zona de estudio. Período 1970-2010 
máximos y mínimos corresponden a valores de $2058 \mathrm{~mm}$ y $782 \mathrm{~mm}$ respectivamente, con una media de $1140 \mathrm{~mm}$ y un desvío estándar de $160 \mathrm{~mm}$. La Figura 10 presenta, mediante un gráfico Box-Plot, la representación estadística de la serie de ETP anuales para el periodo 1970-2010.

En cuanto a la variación estacional, la Figura 11 muestra las fluctuaciones de cada mes para la serie de 40 años, considerando

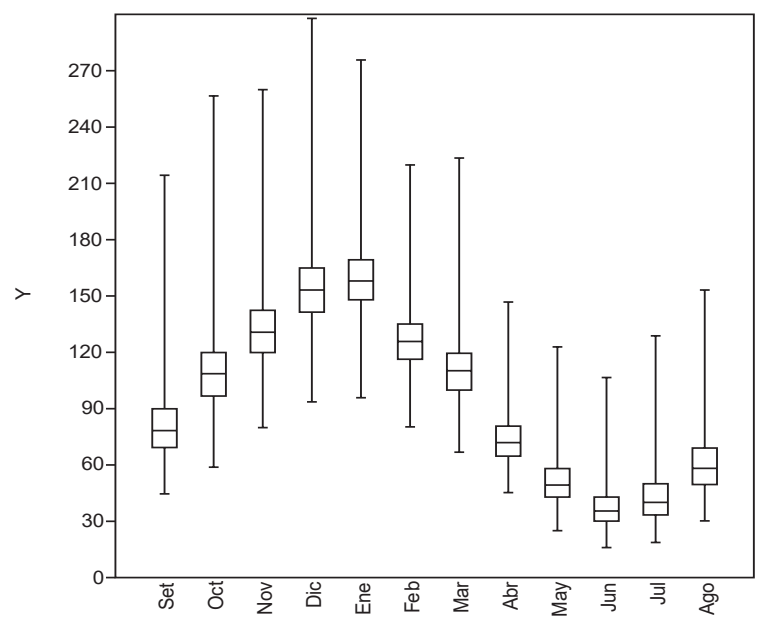

Fig. 10. ETP anual. Período 1970-2010.

Determinar la ETP en sitios puntuales y derivar de ellos un mapa de ETP sirve de base para conocer el estado actual de ésta componente hidrológica. El aumento, o disminución de la ETP, para escenarios futuros, deberá ser considerado en la administración y aprovechamiento de los recursos hídricos, fundamentalmente para fines agrícolas, industrial y urbano.

Los resultados de este trabajo coinciden con lo presentado por el Instituto Nacional de Tecnología Agropecuaria (INTA) que en 2010 confeccionó mapas digitales de precipitación y evapotranspiración potencial (ETP) promedios para cada mes y promedios anuales de una serie de 40 años de registros (1960-2000). todas las estaciones, resultando como es lógico, los mayores valores de ETP en verano y los menores en inviernos con máximas y mínimas del orden de los $270 \mathrm{~mm}$ y $20 \mathrm{~mm}$ respectivamente y un valor medio de $95 \mathrm{~mm}$.

Son los análisis anteriores temporales, ya que se consideran conjuntamente todas las estaciones, independientemente de su ubicación.

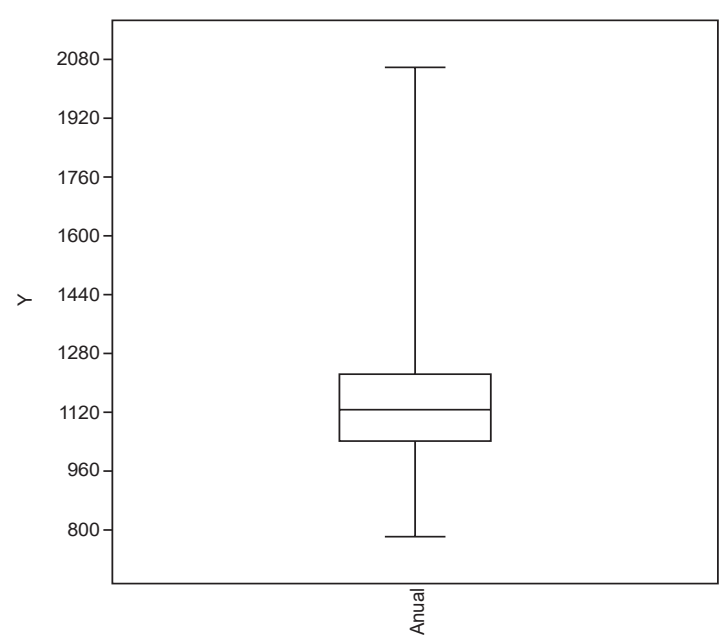

Fig. 11. ETP mensual. Período 1970-2010.

De acuerdo con esa información al restar los mapas anuales de P y ETP se desprende el mapa de balance hídrico directo, mostrado en la Figura 12. En el mismo se observa que la porción noreste del país y una franja próxima a la cordillera, tiene valores positivos, resultando mayor la precipitación que la evapotranspiración potencial en términos anuales. Por el contrario en el Chaco Seco, que es el área correspondiente al oeste de las provincias de Formosa y Chaco, este de Salta y la totalidad de Santiago del Estero, la ETP supera a la $\mathrm{P}$, resultando deficitaria en lo que a volúmenes en términos anuales se analiza. 


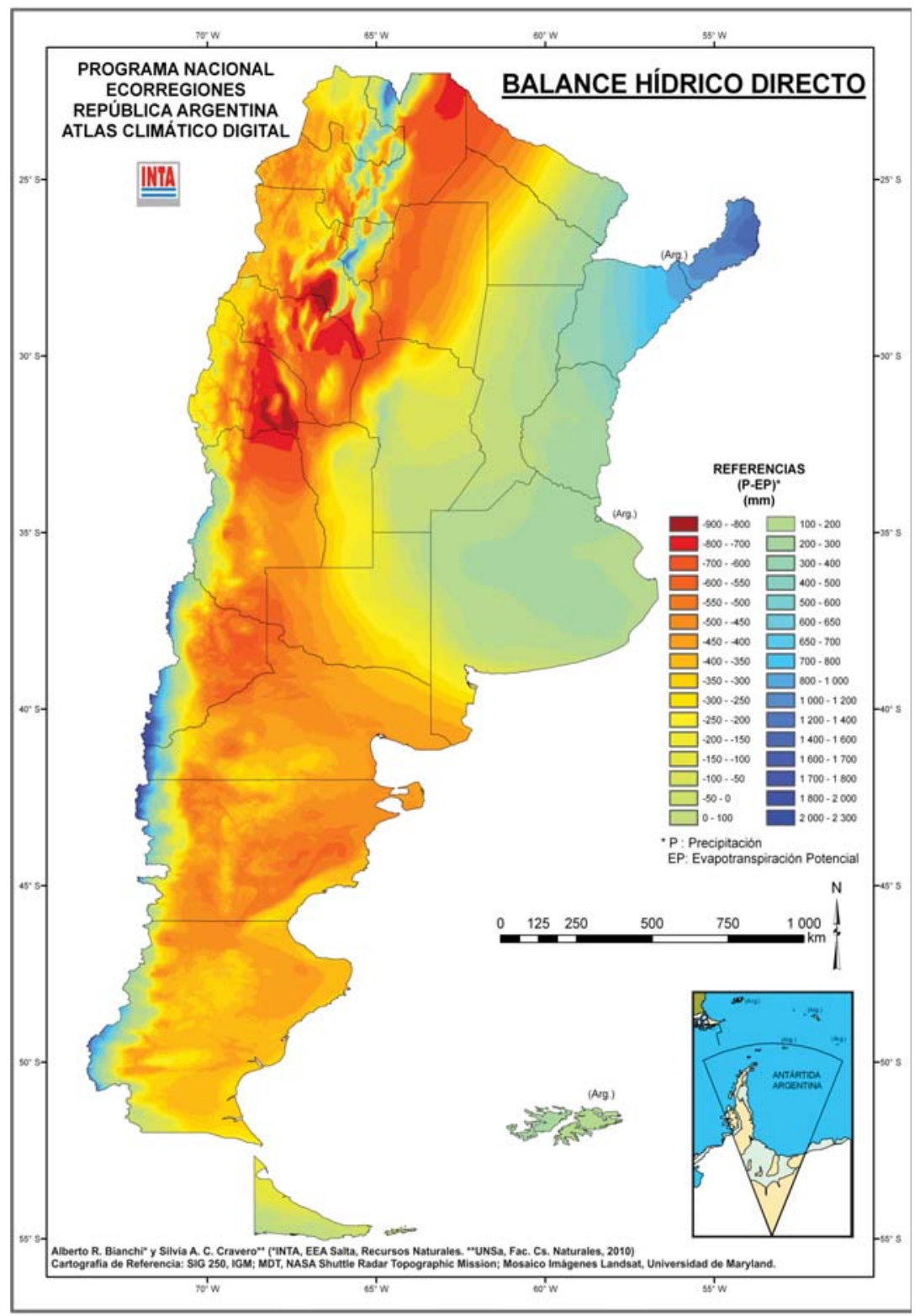

Fig. 12. Balance hídrico en Rep. Argentina. (fuente: INTA, 2010)

CONCLUSIONES I OBSERVACIONES FINALES

1. Se analizaron, para el sector argentino de Cuenca del Plata, la disponibilidad de datos y las lagunas de información en series de datos mensuales de precipitación y de variables meteorológicos durante el período 1970-2010, a partir de la información proporcionada por la Subsecretaria de los Recursos Hídricos de la Nación (SSRH), el Servicio Meteorológico Nacional (SMN), y el Instituto Nacional de Tecnología Agropecuaria (INTA). Tras el relleno de datos faltantes, se pudo disponer, de modo confiable, de 94 estaciones pluviométricas y 38 estaciones con datos para esti- 
mar la evapotranspiración.

2. Del análisis realizado se desprende, que en la mayor parte de la región se observa una tendencia al aumento de precipitación anual desde los años $70 \mathrm{u}$ 80 hasta el 2000 y una leve tendencia a la disminución hacia el año 2010. Pero este análisis es exclusivamente referido al período 1970 - 2010 y no hace ninguna comparación con períodos anteriores. El régimen de precipitaciones anuales presenta una tendencia a agrupar años húmedos y años secos en períodos de entre 3 a 5 años sucesivos, que se intercalan y alternan entre sí. Estos agrupamientos no son homogéneos para toda la región, pero sí se encuentra correspondencia entre las estaciones más próximas.

3. En cuanto a la distribución espacial de la precipitación, el gradiente va aumentando de este a oeste, siendo en la mitad superior de la cuenca, la precipitación anual media de la serie 1970-2010, del orden de los $2200 \mathrm{~mm}$ en Misiones hasta $700 \mathrm{~mm}$ en el este de Formosa y el Chaco, así como en gran parte de Santiago del Estero; e incluso hacia el sur, valores inferiores a $600 \mathrm{~mm}$ anuales. Luego, más hacia al noroeste, debido a la barrera orográfica de la precordillera de los Andes, vuelven a aumentar hasta máximos de $1600 \mathrm{~mm}$. En tanto, para la mitad inferior de la cuenca las precipitaciones anuales varían entre 1400 en el sur de Corrientes, hasta $800 \mathrm{~mm}$ en algunas zonas del oeste de Santa Fe, e incluso $700 \mathrm{~mm}$.

4. La evapotranspiración potencial anual media de la serie 1970-2010, aumenta desde el sureste al noroeste con valores que van desde los $1000 \mathrm{~mm}$, en la pro- vincia de Buenos Aires, hasta $1700 \mathrm{~mm}$ en Santiago del Estero y Salta. Sin embargo en las zonas con relieve más elevado del oeste de Salta y en Jujuy la ETP tiende a disminuir, hasta cifras del orden de $1300 \mathrm{~mm}$.

5. Aunque los máximos de precipitación y los mínimos de ETP no se superponen totalmente, en términos generales, en las zonas de llanura, las precipitaciones más elevadas y las evapotranspiraciones más bajas corresponden al este de la Cuenca del Plata, en donde a escala anual se aprecia que $\mathrm{P}>$ ETP. La aridez presenta tendencia a incrementarse hacia el oeste, de modo que buena parte de Formosa, Chaco, Santiago del Estero y la zona más oriental de Salta, es decir el llamado "Chaco Seco" presentan importantes déficits hídricos.

6. El uso que se haga con esta información se reflejará en los resultados de las políticas locales que se implementen, pero no disponer de ella podrá condenar a la población a una inadecuada y hasta injusta asignación de recursos. Por lo tanto se puede asegurar que el conocimiento de las disponibilidades hídricas en tiempo y en espacio brinda a gestores y planificadores de los recursos hídricos la posibilidad de asignar el recurso de manera eficiente y equitativa, de manera de alcanzar los objetivos de equidad social, sustentabilidad ecológica y eficiencia económica, que promueve la gestión integrada de recursos hídricos.

\section{REFERENCIAS}

Arnold, J.G.; Kiriny, J. R.; Sirinivasan, R.; Williams, J.R.: Haney, E.B.; Neitsch, S.L. (2011) Soil and Water Assessment Tool. 
Input/Ouput Documentation. Version 2012. Technical Report $N^{\circ}$ 439. Texas. Texas Water Resources Institute, 654 pp.

Berbery, E.H.; Barros, V.R. (2002) The hydrologic cycle of the La Plata basin in South America. Journal of Hydrometeorology, 3(6), 630-645.

CEDEX (2003)a Manual de Usuario CHAC. Madrid, España.

CEDEX (2003)b Módulo de estadística aplicada a la Hidrología. Apuntes del Máster de Hidrología Superficial. Publicación interna, $140 \mathrm{pp}$.

Collischonn, W. ; Tucci, C.E.M. (2001) Simulação hidrológica de grandes bacias. Revista Brasileira de Recursos Hídricos, v. 6, n.2.

Collischonn, W. ; Lopes Da Silveira A.L.; Rógenes Monteiro Pontes, P.; Mainardi Fan, F.; Bravo, J.M.; Cauduro Dias De Paiva; R. (2015) Impacto das mudanças climáticas na Bacia do Prata. Produto 2. Projeto: Modelagem Hidrológica da Bacia do Prata Utilizando O Modelo Hidrológico de Grandes Bacias - MGBIPH. Instituto de Pesquisas Hidráulicas (IPH). Porto Alegre, Brasil. 105 p.

Hämmerly, R. (2012) Listado de información nacional potencialmente disponible para el balance hídrico a nivel Cuenca del Plata (Argentina). Informe Final Subcomponente II.1 Balance Hídrico Integrado. Contrato OEA. Santa Fe, Argentina. 75p.

Hämmerly R.; Cacik, P.; Iturraspe, R.; Gagliardi, M.P. (2014) Balance hídrico superficial de la Cuenca del Plata en territorio argentino. Parte $\mathrm{A}$ del Informe Final para el Subcomponente II.1. Balance Hídrico Integrado de la Cuenca del Plata, Comité Intergubernamental Coordinador de los países que conforman la Cuenca del Plata - FICH-UNL. Inédito. 272 pp. Santa Fe, Argentina.

Hämmerly, R.; Cacik, P.; Ingaramo, R.; Paoli, C. (2016) El uso de modelación a paso de tiempo mensual en cuencas no aforadas para el balance hídrico superficial de la Cuenca del Plata. XXVII Congreso Latinoamericano de Hidráulica, Lima, Perú, 28 al 30 de septiembre de 2016.

INTA (2010) Atlas climático digital de la República Argentina. Editores: Bianchi Alberto, Rubí Cravero y Silvia Ana Carla. Salta, Argentina.

Krepper, C.M.; García, N.O.; Jones, P.D. (2008) Low-frequency response of the upper Paraná basin. International Journal of Climatology, 28, 351-360. DOI: 10.1002/joc. 1535.

Paoli C.; Schreider, M. (Ed.) (2000) El río Paraná en su tramo medio. Contribución al conocimiento y prácticas ingenieriles en un gran río de llanura. Centro de Publicaciones UNL. Tomo 1. ISBN: 987508-121-3. 309 pp.

Pereira, L.S.; Teodoro, P.R.; Rodrigues, P.N.; Teixeira, J.L. (2003) Irrigation scheduling simulation: the model ISAREG. En: Tools for Drought Mitigation in Mediterranean Regions, Rossi G., Cancelliere A., Pereira L.S., Oweis T., Shatanawi M., Zairi A. (Eds.). Kluwer, Dordrecht, pp. 161-180.

Tucci, C.E.M.; Clarke, R.T. (1998) Environmental Issues of La Plata Basin. Water Resources Development. v.14, n.2, p.157-173.

USACE (2000). Hydrologic Modeling System HEC-HMS, Technical Reference Manual. US Army Corps of Engineers - Hydrologic Engineering Center. CPD75B. 148 p. 


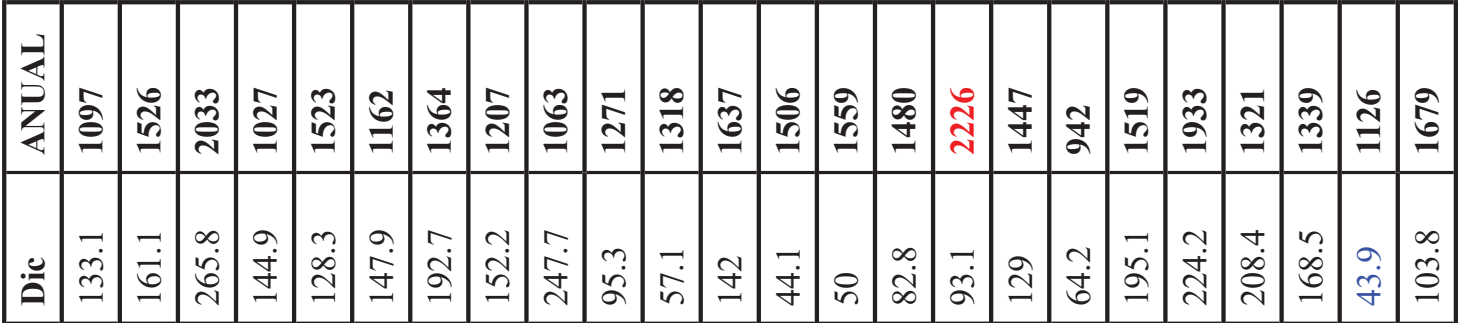

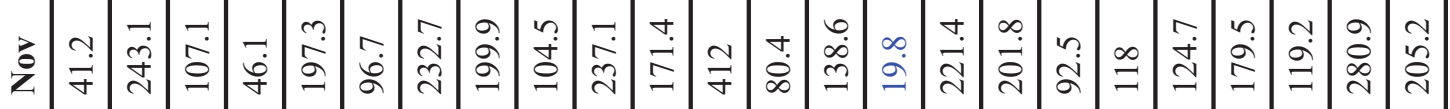

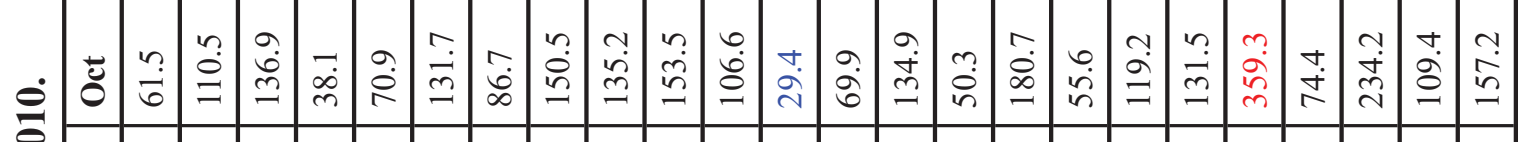

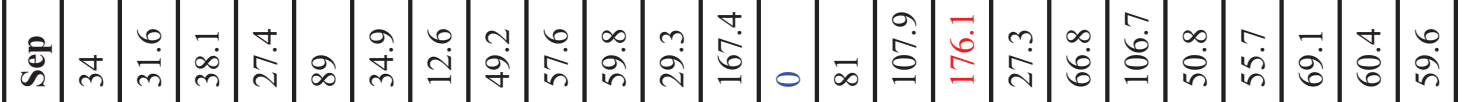

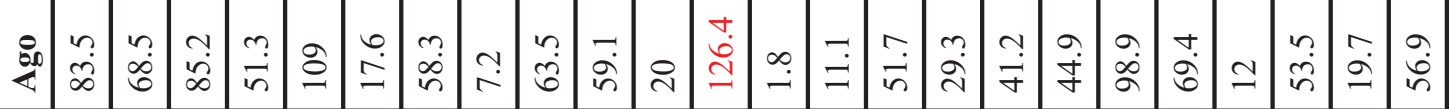




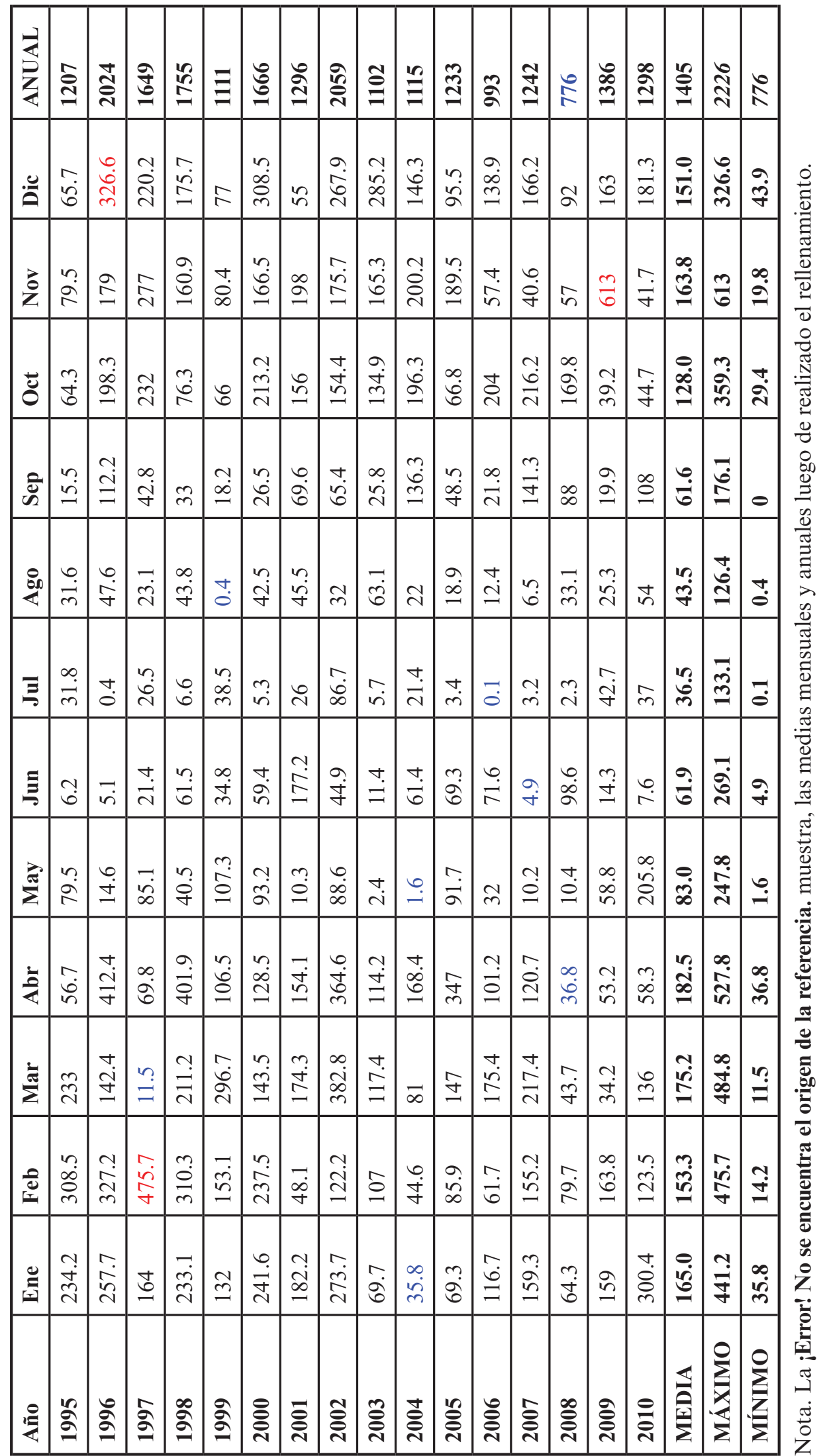




\begin{tabular}{|c|c|c|c|c|c|c|c|c|c|c|c|c|c|c|c|c|c|c|c|c|c|c|c|}
\hline $\begin{array}{l}\sim \\
\infty \\
\infty \\
\infty\end{array}$ & 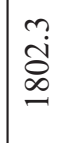 & $\begin{array}{l}n \\
\infty \\
\stackrel{\infty}{2}\end{array}$ & $\begin{array}{l}0 \\
\infty \\
0 \\
\dot{0} \\
+\end{array}$ & $\begin{array}{l}\text { స. } \\
\text { ஸn }\end{array}$ & $\begin{array}{l}\infty \\
0 \\
0 \\
0\end{array}$ & $\begin{array}{l}n \\
8 \\
8\end{array}$ & $\stackrel{n}{\exists}$ & $\begin{array}{l}\stackrel{2}{2} \\
\stackrel{n}{n}\end{array}$ & $\begin{array}{l}\overrightarrow{0} \\
\stackrel{8}{0} \\
\text { In }\end{array}$ & $\begin{array}{l}n \\
0 \\
0 \\
n\end{array}$ & $\begin{array}{l}a \\
\dot{\pi} \\
\tilde{m}\end{array}$ & $\begin{array}{l}\infty \\
\dot{\alpha} \\
\dot{\Xi}\end{array}$ & 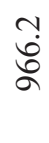 & $\begin{array}{l}\hat{\jmath} \\
\hat{O}\end{array}$ & $\begin{array}{l}-1 \\
0 \\
0 \\
0\end{array}$ & $\begin{array}{l}\mathfrak{n} \\
\varrho\end{array}$ & $\begin{array}{l}0 \\
\stackrel{\sim}{N} \\
\end{array}$ & $\begin{array}{l}\underset{0}{\forall} \\
\stackrel{0}{=}\end{array}$ & $\begin{array}{l}\stackrel{g}{\mathcal{J}} \\
\Xi\end{array}$ & $\begin{array}{l}0 \\
0 \\
0 \\
0\end{array}$ & $\begin{array}{l}n \\
0 \\
0 \\
0\end{array}$ & $\begin{array}{l}+ \\
\text { ஓे }\end{array}$ & $\begin{array}{l}\text { N } \\
\infty \\
\infty \\
\infty\end{array}$ \\
\hline$\stackrel{0}{=}$ & $\hat{8}$ & O̊. & $\frac{0}{n}$ & $\stackrel{\vartheta}{\dot{f}}$ & $\stackrel{0}{\stackrel{\sim}{0}}$ & 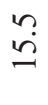 & $\stackrel{n}{\ddot{\gamma}}$ & $\vec{\infty}$ & $\stackrel{n}{\infty}$ & $\stackrel{9}{\circ}$ & 훙 & $\begin{array}{l}\infty \\
\dot{b}\end{array}$ & $\stackrel{\infty}{\dot{\Xi}}$ & $\stackrel{n}{\stackrel{n}{2}}$ & $\frac{m}{m}$ & $\stackrel{n}{n}$ & $\vec{n}$ & $\vec{g}$ & $\begin{array}{l}n \\
\tilde{n}\end{array}$ & $\frac{n}{n}$ & $\vec{b}$ & $\begin{array}{l}\infty \\
\stackrel{\infty}{2}\end{array}$ & 芦 \\
\hline$\Xi$ & $\because$ & $\ddot{\ddot{g}}$ & $\frac{\infty}{\dot{\gamma}}$ & $\begin{array}{l}0 \\
\dot{0}\end{array}$ & $\stackrel{\infty}{\stackrel{\infty}{\Sigma}}$ & $\stackrel{\sim}{ \pm}$ & ñ & na & ָे & $\begin{array}{l}0 \\
\infty \\
\infty\end{array}$ & $\begin{array}{l}0 \\
0 \\
0\end{array}$ & $\underset{⿱}{\stackrel{+}{+}}$ & $\underset{\dot{J}}{+}$ & $\begin{array}{l}0 \\
\stackrel{0}{2}\end{array}$ & 守 & $\tilde{\curvearrowright}$ & $\vec{b}$ & $\begin{array}{l}\infty \\
\stackrel{+}{n}\end{array}$ & 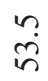 & $\stackrel{\infty}{\sim}$ & $\begin{array}{l}0 \\
\grave{\lambda}\end{array}$ & $\stackrel{\sim}{ָ}$ & ? \\
\hline
\end{tabular}

Z

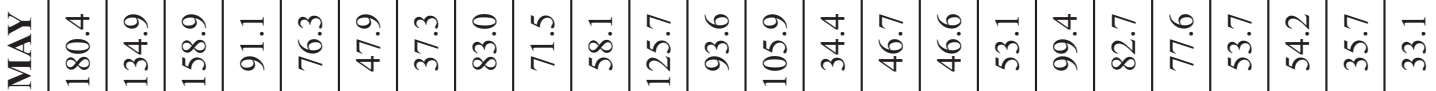

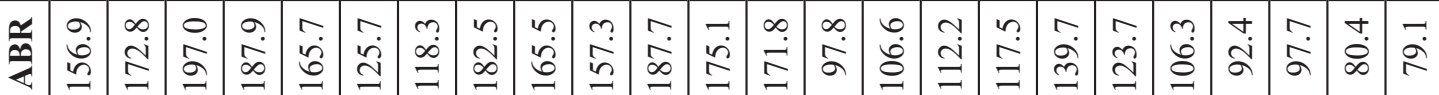
そ) $\leadsto$ అ

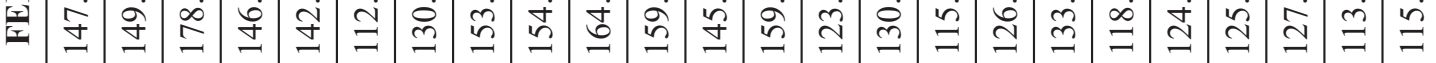

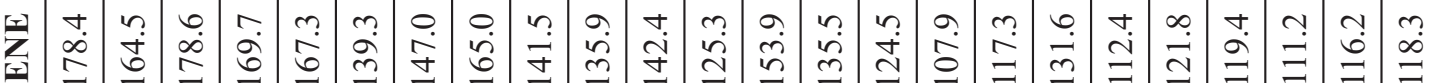

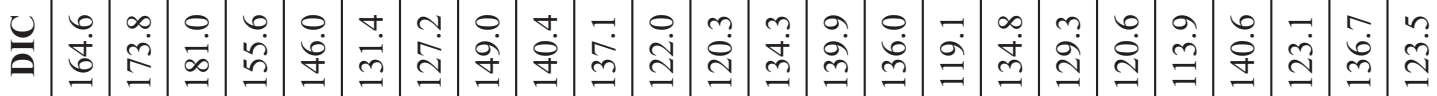

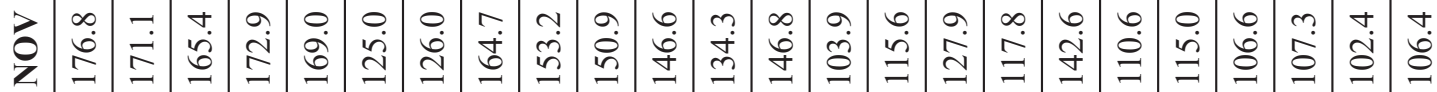
E $\boldsymbol{U}$ ก

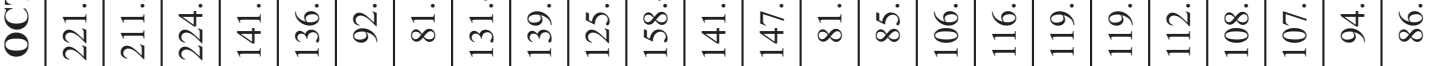

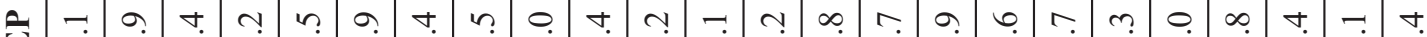

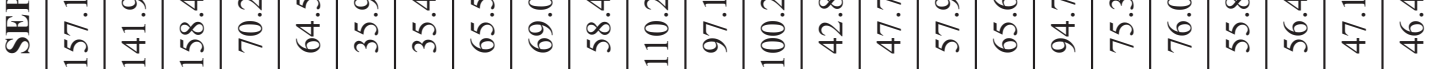




\begin{tabular}{|c|c|c|c|c|c|c|c|c|c|c|c|c|c|c|c|c|c|c|c|c|c|c|c|c|}
\hline & 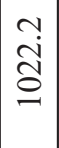 & $\overrightarrow{\tilde{n}}$ & $\begin{array}{l}m \\
\stackrel{2}{\infty} \\
\infty\end{array}$ & $\begin{array}{l}a \\
\bar{n} \\
0\end{array}$ & $\stackrel{\infty}{\dot{0}}$ & $\frac{1}{8}$ & $\begin{array}{l}\hat{\infty} \\
\dot{0} \\
=\end{array}$ & $\begin{array}{l}\stackrel{0}{1} \\
\stackrel{\hat{S}}{0}\end{array}$ & \begin{tabular}{l|}
$\infty$ \\
$\infty$ \\
$\infty$ \\
0 \\
0
\end{tabular} & \begin{tabular}{|c|}
0 \\
$\dot{8}$ \\
0
\end{tabular} & $\begin{array}{l}a \\
\tilde{\hat{\theta}} \\
\hat{o}\end{array}$ & $\begin{array}{l}+ \\
\infty \\
\infty\end{array}$ & $\begin{array}{l}-\vec{a} \\
0 \\
0\end{array}$ & $\begin{array}{l}\overrightarrow{0} \\
\dot{0}\end{array}$ & $\begin{array}{l}0 \\
\dot{J} \\
\infty\end{array}$ & 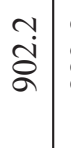 & $\begin{array}{l}\infty \\
i \\
\infty \\
\infty \\
\infty\end{array}$ & \begin{tabular}{l|l}
$\infty$ \\
\\
\\
\end{tabular} & 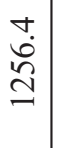 & $\begin{array}{l}n \\
\stackrel{n}{o} \\
=\end{array}$ & 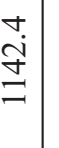 & g. & \multicolumn{2}{|c|}{ 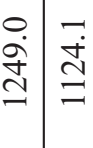 } \\
\hline$m$ & $\begin{array}{l}n \\
\tilde{n} \\
m\end{array}$ & $\vec{n}$ & $\stackrel{\sim}{\circ}$ & \begin{tabular}{l|} 
\\
$\dot{f}$ \\
$\dot{f}$
\end{tabular} & 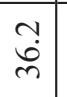 & $\begin{array}{l}\infty \\
\infty \\
a\end{array}$ & $\begin{array}{l}+\dot{b} \\
\qquad\end{array}$ & $\stackrel{+}{\dot{\sigma}}$ & $\begin{array}{l}0 \\
i n \\
\text { in }\end{array}$ & \begin{tabular}{l|}
$a$ \\
$\dot{b}$ \\
in
\end{tabular} & $\begin{array}{l} \\
\\
\text { i }\end{array}$ & $\begin{array}{l}0 \\
0 \\
6\end{array}$ & î́ & ते & $\begin{array}{l}\dot{0} \\
\dot{m}\end{array}$ & 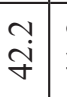 & $\frac{\dot{q}}{\sigma}$ & 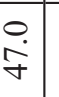 & $\begin{array}{l}\infty \\
\dot{m} \\
\dot{m}\end{array}$ & $\overline{\text { m }}$ & $\widehat{\tilde{N}}$ & $\stackrel{\nabla}{\grave{\lambda}}$ & $\underset{m}{\stackrel{m}{m}}$ & \\
\hline 户े & $\vec{m}$ & $\begin{array}{l}\stackrel{0}{\dot{m}} \\
\text { nat }\end{array}$ & $\stackrel{+}{\dot{\infty}}$ & $\frac{n}{q}$ & $\stackrel{f}{\sim}$ & $\begin{array}{l}\dot{0} \\
\dot{\imath}\end{array}$ & $\overrightarrow{8}$ & $\vec{i}$ & 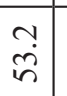 & 点 & ते & $\vec{b}$ & $\begin{array}{l}\infty \\
\infty \\
\infty \\
i\end{array}$ & 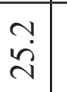 & $\begin{array}{l}0 \\
\dot{m}\end{array}$ & $\overrightarrow{\dot{\gamma}}$ & $\begin{array}{l}\infty \\
\text { m. } \\
\text { m. }\end{array}$ & $\frac{0}{\dot{n}}$ & $\begin{array}{l}n \\
\tilde{\sigma}\end{array}$ & $\overrightarrow{\mathrm{d}}$ & $\begin{array}{l}\stackrel{0}{\vec{v}} \\
\ddot{\sim}\end{array}$ & $\overrightarrow{\ddot{v}}$ & $\stackrel{\infty}{\dot{m}}$ & $\dot{\rho}$ \\
\hline & $\underset{m}{c}$ & 官 & $\stackrel{\rightarrow}{\Omega}$ & $\begin{array}{l}\infty \\
i \\
i\end{array}$ & $\vec{a}$ & $\begin{array}{l}\infty \\
\dot{v} \\
\vec{v}\end{array}$ & $\begin{array}{l}0 \\
\stackrel{0}{8}\end{array}$ & $\begin{array}{l}\text { N } \\
\text { H্ }\end{array}$ & in & $\begin{array}{l}n \\
\text { ñ } \\
\text { in }\end{array}$ & $\begin{array}{l}\stackrel{0}{0} \\
\dot{\infty} \\
\text { in }\end{array}$ & $\underset{\dot{\sigma}}{\dot{\sigma}}$ & $\bar{n}$ & mु. & $\begin{array}{l}\text { ర0 } \\
\dot{m}\end{array}$ & & $n$ & n & 7 & ले & $\begin{array}{l}0 \\
\infty \\
\infty \\
m\end{array}$ & \& & $\stackrel{0}{-}$ & \\
\hline
\end{tabular}

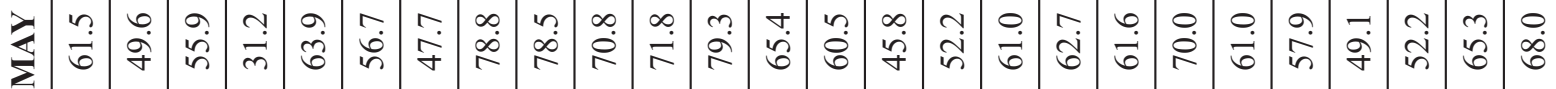

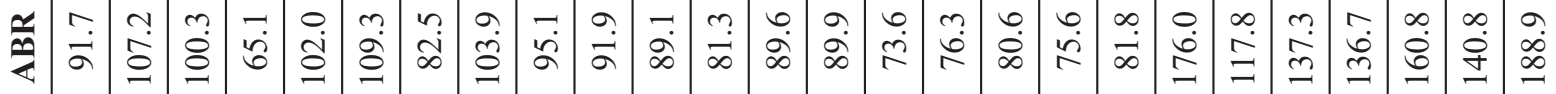

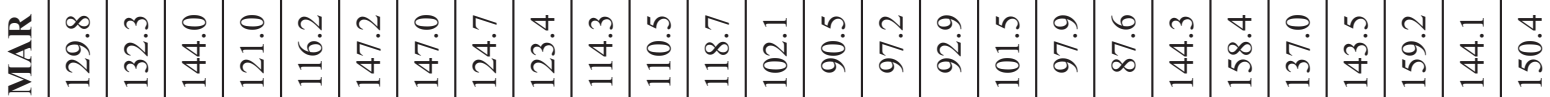

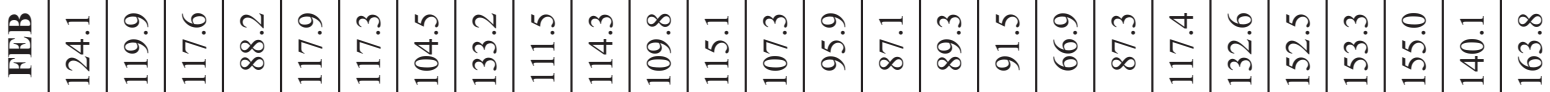

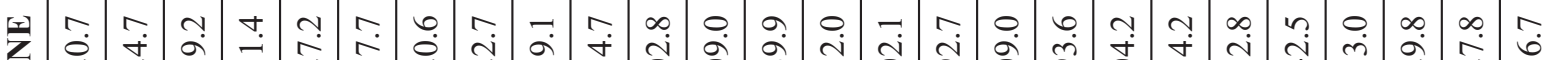

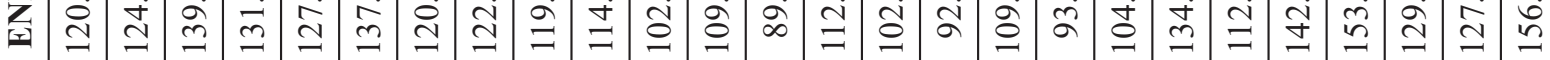

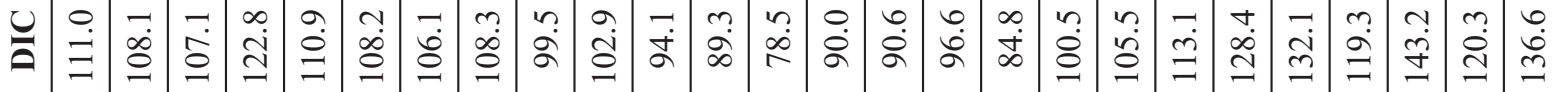

z)

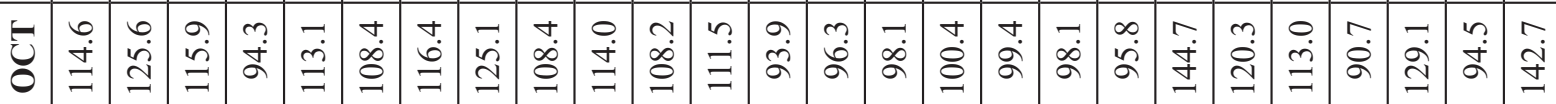

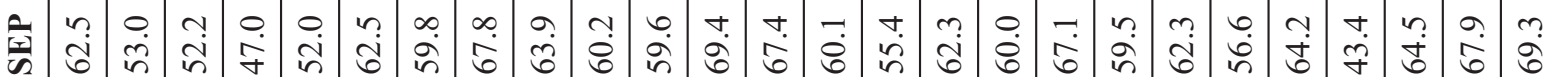

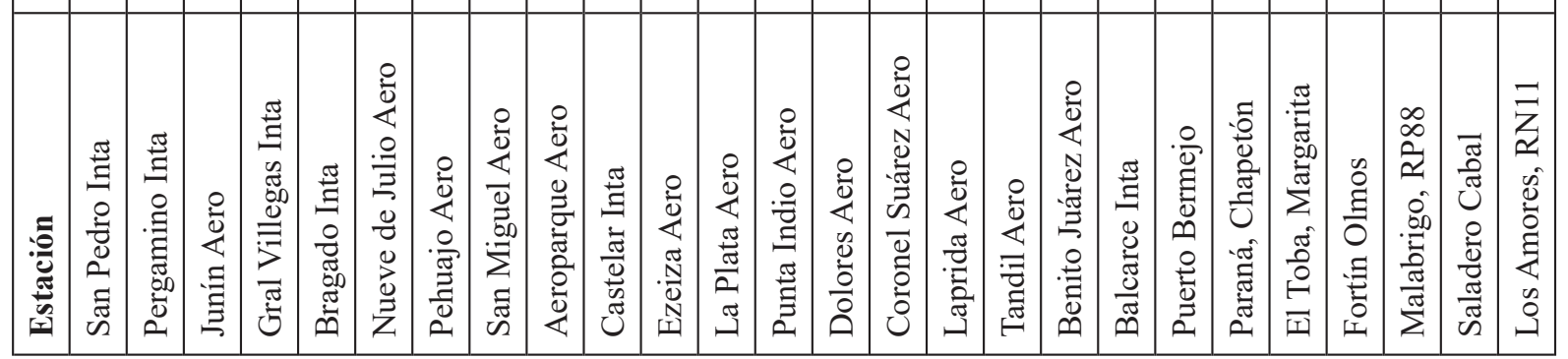




\begin{tabular}{|c|c|c|c|c|c|c|c|c|c|c|c|c|c|c|c|c|c|c|c|c|c|c|c|c|c|}
\hline & \begin{tabular}{|l|}
$\vec{i}$ \\
$\stackrel{+}{ \pm}$ \\
$\beth$
\end{tabular} & \begin{tabular}{|l|}
$n$ \\
$\infty$ \\
$\alpha$ \\
$\alpha$ \\
\end{tabular} & 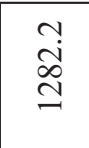 & $\begin{array}{l}0 \\
\stackrel{0}{\tilde{n}} \\
\stackrel{I}{I}\end{array}$ & \begin{tabular}{|l|} 
\\
iे \\
$\tilde{\sigma}$
\end{tabular} & \begin{tabular}{l|} 
\\
$\dot{0}$ \\
$\stackrel{0}{ }$ \\
0
\end{tabular} & $\begin{array}{l}0 \\
\ddot{d} \\
\tilde{0} \\
0\end{array}$ & $\begin{array}{l}\stackrel{\mathfrak{\Xi}}{\mathrm{I}} \\
\stackrel{\Xi}{\Xi}\end{array}$ & \begin{tabular}{|l|} 
\\
$\dot{n}$ \\
$m$ \\
$n$
\end{tabular} & \begin{tabular}{|c|} 
\\
$\dot{w}$ \\
$\dot{+}$ \\
$\infty$ \\
\end{tabular} & $\begin{array}{l}0 \\
\infty \\
\curvearrowright \\
\curvearrowright\end{array}$ & $\begin{array}{l}\overrightarrow{\hat{s}} \\
\stackrel{8}{-}\end{array}$ & $\begin{array}{l}n \\
2 \\
\infty \\
\infty \\
\infty\end{array}$ & 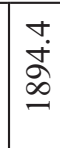 & $\frac{n}{\stackrel{2}{\hat{S}}}$ & 䒘 & $\frac{n}{\tilde{2}}$ & $\begin{array}{l}0 \\
0 \\
\mathbb{b} \\
\infty \\
-\end{array}$ & \begin{tabular}{|l|} 
\\
$\dot{s}$ \\
$\infty$ \\
$\Xi$
\end{tabular} & 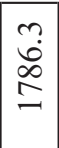 & \begin{tabular}{|l|} 
\\
$n$ \\
$b$ \\
$n$ \\
$n$ \\
$n$
\end{tabular} & 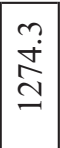 & 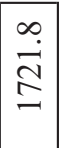 & $\begin{array}{l}0 \\
\ddot{n} \\
\ddot{n} \\
n\end{array}$ & $\begin{array}{l}a \\
\stackrel{0}{0} \\
\text { ปे }\end{array}$ \\
\hline & $\begin{array}{r}\nabla \\
\ddot{v}\end{array}$ & $\frac{m}{m}$ & ñ & $\mid \begin{array}{l}\infty \\
\text { i }\end{array}$ & $\begin{array}{l}n \\
\infty \\
n\end{array}$ & $\begin{array}{c}n \\
\infty \\
m\end{array}$ & $\begin{array}{l}\dot{0} \\
\vec{\sim}\end{array}$ & $\begin{array}{l}\infty \\
\stackrel{\infty}{ } \\
\infty\end{array}$ & $\begin{array}{l}\hat{n} \\
\dot{n}\end{array}$ & \begin{tabular}{|l|} 
\\
$\stackrel{0}{0}$ \\
$=$
\end{tabular} & $\stackrel{\circ}{\stackrel{0}{0}}$ & $\mid \begin{array}{l}\infty \\
\stackrel{0}{1} \\
\end{array}$ & $\hat{a}$ & $\underset{\stackrel{g}{I}}{\mathrm{~g}}$ & \begin{tabular}{|l|} 
\\
$\dot{g}$ \\
$\dot{g}$
\end{tabular} & $\begin{array}{l}n \\
\tilde{n} \\
\end{array}$ & $2 n$ & $\begin{array}{l} \\
\\
0 \\
\end{array}$ & 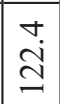 & $\begin{array}{l}0 \\
\dot{0} \\
0\end{array}$ & \begin{tabular}{|l|} 
\\
$\dot{+}$ \\
\end{tabular} & \begin{tabular}{|l|}
$\dot{a}$ \\
$\infty$ \\
$\infty$
\end{tabular} & $\begin{array}{l} \\
\dot{0} \\
\end{array}$ & $\begin{array}{l}n \\
n \\
i n\end{array}$ & $\begin{array}{l}\infty \\
\dot{b} \\
\dot{+}\end{array}$ \\
\hline & \begin{tabular}{|l|}
$\infty$ \\
$\grave{\lambda}$ \\
$\dot{\lambda}$
\end{tabular} & \begin{tabular}{l|} 
\\
$\dot{i}$ \\
|
\end{tabular} & $\frac{n}{m}$ & rn & $\stackrel{+}{\stackrel{\sim}{\sim}}$ & $\stackrel{a}{\hat{\imath}}$ & $\stackrel{\overbrace{}}{\dot{\sim}}$ & $\begin{array}{l}n \\
\stackrel{n}{n}\end{array}$ & $\stackrel{+}{\grave{\lambda}}$ & $\begin{array}{l}0 \\
\dot{8} \\
0\end{array}$ & $\stackrel{\widehat{\Xi}}{\Xi}$ & $\begin{array}{l}\infty \\
\hat{\alpha} \\
\end{array}$ & $\stackrel{\circ}{\stackrel{0}{=}}$ & $\underset{\Xi}{\Xi}$ & ñ & $\overrightarrow{\check{a}}$ & $\begin{array}{l}0 \\
\stackrel{0}{2}\end{array}$ & $\tilde{a}$ & $\mid \begin{array}{l}a \\
\dot{0} \\
\text { I }\end{array}$ & $\begin{array}{l}+ \\
\dot{0}\end{array}$ & $\frac{a}{i}$ & $\begin{array}{l}\hat{j} \\
\dot{m} \\
\dot{n}\end{array}$ & $\hat{\tilde{o}}$ & $\begin{array}{l}\infty \\
i \\
i\end{array}$ & $\begin{array}{l}0 \\
i n\end{array}$ \\
\hline & $\stackrel{?}{\stackrel{f}{f}}$ & $\begin{array}{c}\tilde{n} \\
\tilde{n}\end{array}$ & $\stackrel{\check{F}}{\stackrel{f}{f}}$ & $\begin{array}{l}0 \\
\dot{J} \\
\dot{f}\end{array}$ & $\vec{b}$ & 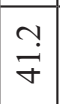 & $\begin{array}{l}0 \\
\dot{m} \\
\dot{m}\end{array}$ & $\frac{a}{\vec{n}}$ & $\overrightarrow{0}$ & $\overrightarrow{ \pm}$ & 守 & $\begin{array}{l}\infty \\
\dot{g} \\
\end{array}$ & $\frac{a}{\vec{n}}$ & $\underset{\text { in }}{\stackrel{+}{n}}$ & $\stackrel{\circ}{\stackrel{2}{2}}$ & ñ & $\begin{array}{l}\overrightarrow{0} \\
n\end{array}$ & $\overrightarrow{\dot{0}}$ & $\begin{array}{l}0 \\
\dot{0} \\
\dot{+} \\
\end{array}$ & $\begin{array}{l}a \\
\grave{\omega}\end{array}$ & $\begin{array}{l}\mathscr{J} \\
\Xi \\
\Xi\end{array}$ & $\begin{array}{l}+ \\
\dot{0} \\
+\end{array}$ & $\stackrel{\vec{g}}{\stackrel{\oplus}{\Xi}}$ & 官 & $\stackrel{0}{2}$ \\
\hline & $\frac{m}{i n}$ & $\begin{array}{l}0 \\
\dot{\gamma} \\
\dot{\gamma}\end{array}$ & $\vec{n}$ & $\frac{n}{n}$ & in & $\ddot{\bullet}$ & $\begin{array}{l}\hat{n} \\
\tilde{n}\end{array}$ & $\begin{array}{c}m \\
\stackrel{n}{n}\end{array}$ & $\mid \begin{array}{c}n \\
\infty \\
0 \\
0\end{array}$ & $\mid \begin{array}{c}a \\
\hat{2} \\
-\end{array}$ & 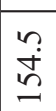 & $\begin{array}{l}n \\
\stackrel{2}{n} \\
\sim\end{array}$ & $\stackrel{0}{a}$ & 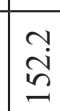 & $\begin{array}{l}0 \\
\infty \\
\stackrel{0}{\Xi}\end{array}$ & $\begin{array}{l}n \\
\stackrel{2}{2}\end{array}$ & $\stackrel{\infty}{\infty}$ & $\hat{\sim}$ & $\frac{\infty}{i}$ & $\begin{array}{l}\grave{a} \\
\grave{a}\end{array}$ & $\stackrel{n}{i}$ & 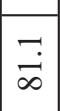 & $\begin{array}{l}\forall \\
\dot{0} \\
\dot{J}\end{array}$ & $\underset{\infty}{\infty}$ & $\infty$ \\
\hline & $\begin{array}{c}m \\
n \\
n\end{array}$ & $\hat{\sigma}$ & o̊ & 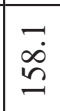 & $\begin{array}{l}0 \\
\dot{0} \\
\infty\end{array}$ & $\begin{array}{l}r \\
n \\
n\end{array}$ & $\begin{array}{l}n \\
\\
\simeq\end{array}$ & \begin{tabular}{l}
$\mathfrak{y}$ \\
$\dot{v}$ \\
\cline { 1 - 1 }
\end{tabular} & $\stackrel{2}{\Omega}$ & $\mid \begin{array}{c}n \\
\infty \\
0 \\
0 \\
-1\end{array}$ & 光 & \begin{tabular}{|l|} 
\\
0 \\
0 \\
0
\end{tabular} & $\frac{n}{\infty}$ & $\begin{array}{l}+ \\
\infty \\
\infty \\
0 \\
-\end{array}$ & $\begin{array}{l}n \\
0 \\
\infty \\
-1\end{array}$ & $\begin{array}{l}n \\
\stackrel{n}{0} \\
=\end{array}$ & $\underset{\sim}{\sim}$ & ํ. & $\begin{array}{l}\infty \\
i \\
\sim \\
\sim\end{array}$ & $\stackrel{\sim}{\dot{J}}$ & $\begin{array}{l}\tilde{i} \\
\stackrel{2}{2}\end{array}$ & \begin{tabular}{|l|}
$\infty$ \\
$\dot{J}$ \\
- \\
\end{tabular} & $\frac{\vec{\pi}}{\sim}$ & 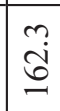 & $\underset{\dot{n}}{\infty}$ \\
\hline & $\vec{~} \vec{i}$ & 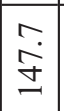 & $\begin{array}{l}\overrightarrow{0} \\
\infty \\
\infty\end{array}$ & $\stackrel{n}{i}$ & $\stackrel{\stackrel{\vartheta}{m}}{=}$ & ले & 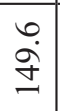 & $\stackrel{\text { 妾 }}{=}$ & $\begin{array}{l}0 \\
\dot{1} \\
\end{array}$ & 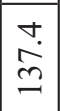 & $\stackrel{0}{0}$ & $\begin{array}{l}n \\
\tilde{m} \\
\end{array}$ & $\begin{array}{l}0 \\
\stackrel{1}{I} \\
\end{array}$ & ํ. & $\frac{n}{\dot{z}}$ & 㢳 & $\begin{array}{l}0 \\
\dot{0} \\
0\end{array}$ & $\stackrel{2}{\stackrel{2}{n}}$ & $\stackrel{\circ}{\stackrel{1}{I}}$ & $\stackrel{\sim}{\stackrel{\sim}{\Xi}}$ & \begin{tabular}{|l|}
\multirow{2}{*}{} \\
$\infty$ \\
$\infty$ \\
0 \\
\end{tabular} & $\begin{array}{l}0 \\
\hat{\sigma} \\
-\end{array}$ & 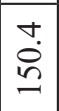 & $\begin{array}{l}0 \\
\dot{m} \\
\ddot{n}\end{array}$ & $\stackrel{\text { บ }}{n}$ \\
\hline & $\begin{array}{l}\infty \\
\dot{\Omega} \\
\end{array}$ & $\begin{array}{l}+ \\
\dot{8} \\
\end{array}$ & 点 & 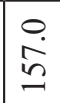 & $\stackrel{\overrightarrow{6}}{=}$ & $\begin{array}{l}0 \\
\dot{0} \\
\end{array}$ & $\begin{array}{l}\stackrel{0}{\mathscr{D}} \\
\stackrel{\sim}{ \pm}\end{array}$ & $\stackrel{\vartheta}{\tilde{\Xi}}$ & 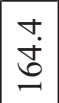 & \begin{tabular}{|c|}
$\vec{\infty}$ \\
$\dot{0}$ \\
\end{tabular} & \begin{tabular}{|l|}
$\infty$ \\
$\dot{0}$ \\
0
\end{tabular} & $\begin{array}{l}0 \\
\dot{0} \\
2\end{array}$ & $\begin{array}{l}t \\
\dot{8} \\
\ddot{n}\end{array}$ & $\begin{array}{l}0 \\
\dot{J}\end{array}$ & 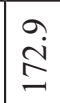 & $\frac{9}{2}$ & $\vec{g}$ & $\begin{array}{l}0 \\
i \\
i \\
\end{array}$ & $\begin{array}{l}\infty \\
\dot{\mathfrak{b}} \\
\end{array}$ & $\begin{array}{l}\stackrel{\sim}{n} \\
\tilde{n} \\
n\end{array}$ & $\begin{array}{l}\overrightarrow{\dot{D}} \\
\dot{m}\end{array}$ & \begin{tabular}{|l|} 
\\
$\dot{g}$ \\
$\dot{g}$
\end{tabular} & \begin{tabular}{|l|} 
\\
$i$ \\
$\mathcal{J}$
\end{tabular} & $\frac{0}{\dot{m}}$ & $\vec{m}$ \\
\hline & $\mid \begin{array}{l}n \\
\infty \\
+ \\
+\end{array}$ & 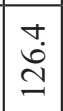 & $\stackrel{\stackrel{3}{I}}{I}$ & $\begin{array}{l}n \\
\mathfrak{y} \\
\dot{J}\end{array}$ & $\stackrel{1}{8}$ & $\begin{array}{l}\dot{0} \\
\dot{m}\end{array}$ & $\begin{array}{l}\infty \\
\underset{\Xi}{\Xi}\end{array}$ & $\stackrel{n}{\stackrel{n}{\beth}}$ & $\stackrel{0}{8}$ & $\begin{array}{l}\vec{\infty} \\
\stackrel{\infty}{n} \\
\end{array}$ & $\frac{n}{6}$ & $\begin{array}{l}0 \\
\stackrel{0}{ } \\
\text { in }\end{array}$ & $\stackrel{\infty}{\stackrel{\infty}{I}}$ & $\frac{\mathfrak{r}}{\tilde{6}}$ & 守 & $\begin{array}{l}n \\
\stackrel{\infty}{\infty} \\
\end{array}$ & $\begin{array}{l}\infty \\
\dot{b} \\
0\end{array}$ & $\stackrel{\infty}{\check{\check{n}}}$ & $\begin{array}{l}0 \\
\stackrel{2}{2}\end{array}$ & $\stackrel{\substack{\infty \\
\Sigma}}{\stackrel{\infty}{\Sigma}}$ & 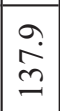 & \begin{tabular}{|l|}
$\infty$ \\
$\stackrel{\dot{q}}{ \pm}$ \\
\end{tabular} & \begin{tabular}{|l|} 
\\
$\infty$ \\
$\dot{0}$ \\
\end{tabular} & $\stackrel{\mathscr{m}}{\stackrel{\Xi}{\Xi}}$ & ت્ \\
\hline & $\frac{i}{m}$ & 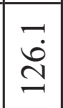 & $\stackrel{\sim}{\Omega}$ & 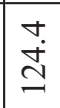 & $\overrightarrow{\stackrel{M}{\Xi}}$ & $\dot{m}$ & $\stackrel{\vec{\Xi}}{=}$ & $\begin{array}{l}\infty \\
\stackrel{0}{0} \\
\stackrel{0}{-}\end{array}$ & $\begin{array}{l}0 \\
\\
n \\
-1\end{array}$ & $\frac{n}{\Sigma}$ & $\ddot{\sigma}$ & $\begin{array}{l}\vec{\infty} \\
\stackrel{\infty}{n}\end{array}$ & $\underset{\dot{\sigma}}{+}$ & 守 & 莳 & $\stackrel{\sim}{\sigma}$ & 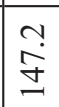 & $\begin{array}{l}n \\
\stackrel{2}{n} \\
\end{array}$ & \begin{tabular}{|l}
$\infty$ \\
$\stackrel{\sim}{+}$ \\
\multirow{J}{*}{}
\end{tabular} & 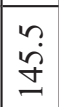 & $\begin{array}{l}\infty \\
\dot{m} \\
\sim\end{array}$ & $\begin{array}{l}\vec{j} \\
\stackrel{\sim}{\sim}\end{array}$ & $\begin{array}{l}m \\
\infty \\
0 \\
\beth\end{array}$ & $\begin{array}{l}\text { ゙ָ } \\
\text { ปิ }\end{array}$ & $\stackrel{\circ}{\circ}$ \\
\hline & $\mid \begin{array}{l}+ \\
\dot{0} \\
\dot{+}\end{array}$ & $\begin{array}{l}0 \\
\dot{0} \\
0\end{array}$ & $\begin{array}{l}n \\
\infty \\
\infty \\
n\end{array}$ & $\begin{array}{l}\infty \\
\stackrel{I}{I} \\
\stackrel{1}{I}\end{array}$ & 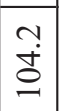 & $\begin{array}{l}0 \\
\stackrel{0}{0}\end{array}$ & $\hat{\grave{d}}$ & $\overrightarrow{0}$ & $\begin{array}{l}\vec{\infty} \\
\infty \\
\end{array}$ & \begin{tabular}{l}
$n$ \\
0 \\
8 \\
\hdashline \\
\end{tabular} & $\begin{array}{l}0 \\
i \\
\infty \\
0\end{array}$ & 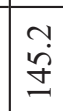 & $\overrightarrow{\dot{\infty}}$ & $\stackrel{\infty}{\stackrel{\infty}{\Sigma}}$ & $\frac{n}{\infty}$ & $\begin{array}{l}\stackrel{i}{i} \\
\stackrel{i}{I}\end{array}$ & $\stackrel{\square}{\stackrel{2}{2}}$ & $\begin{array}{l}\infty \\
\infty \\
\stackrel{\infty}{n}\end{array}$ & $\begin{array}{l}\stackrel{q}{\dot{y}} \\
\dot{\Xi}\end{array}$ & $\begin{array}{l}a \\
\dot{\infty} \\
0 \\
0\end{array}$ & $\begin{array}{l}+ \\
\stackrel{+}{n} \\
\end{array}$ & $\begin{array}{l}m \\
\stackrel{m}{n} \\
\end{array}$ & $\begin{array}{l}\infty \\
\dot{\forall} \\
\end{array}$ & $\begin{array}{l}0 \\
\dot{J} \\
\end{array}$ & $\begin{array}{l} \\
\stackrel{+}{ \pm} \\
\end{array}$ \\
\hline & $\ddot{\circ}$ & $\begin{array}{l}\infty \\
\dot{0} \\
0\end{array}$ & $\stackrel{\varrho}{\Xi}$ & $\begin{array}{l}\infty \\
\\
ٍ\end{array}$ & 吕 & $\begin{array}{l}\stackrel{\nabla}{\bullet} \\
=\end{array}$ & $\frac{0}{\infty}$ & $\stackrel{\infty}{\Xi}$ & \begin{tabular}{|l|}
$a$ \\
$\infty$ \\
$\stackrel{0}{\circlearrowleft}$ \\
\end{tabular} & 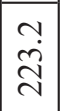 & $\begin{array}{l}0 \\
i \\
\tilde{d}\end{array}$ & 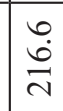 & ते & $\begin{array}{l}a \\
\hat{\infty} \\
\hat{v}\end{array}$ & $\begin{array}{l}0 \\
\stackrel{0}{0} \\
\stackrel{n}{2}\end{array}$ & $\stackrel{\sim}{\infty}$ & $\overrightarrow{\text { ते }}$ & $\vec{a}$ & $\frac{\Im}{\vec{\lambda}}$ & $\dot{\sim}$ & 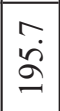 & 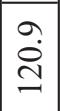 & 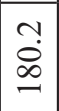 & $\begin{array}{l}a \\
\stackrel{0}{n} \\
-\end{array}$ & 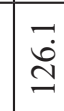 \\
\hline & $\vec{i}$ & $\begin{array}{l}+ \\
\dot{n} \\
i\end{array}$ & ? & in & $\begin{array}{l}0 \\
\dot{f}\end{array}$ & $\stackrel{q}{q}$ & $\vec{\sim}$ & $\underset{n}{\stackrel{r}{n}}$ & $\begin{array}{l}+ \\
\dot{0} \\
\dot{0}\end{array}$ & \begin{tabular}{|l|} 
\\
$\dot{o}$ \\
$\dot{o}$
\end{tabular} & \begin{tabular}{l}
0 \\
\multirow{0}{0}{} \\
-
\end{tabular} & $\begin{array}{l}0 \\
\dot{n} \\
n\end{array}$ & 竞 & $\begin{array}{l}0 \\
\text { in } \\
\end{array}$ & 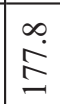 & 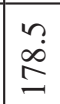 & $\begin{array}{l}\hat{\text { a }} \\
\text { nn }\end{array}$ & $\stackrel{r}{\tilde{n}}$ & $\begin{array}{l}-7 \\
\dot{b} \\
\end{array}$ & $\begin{array}{l}\dot{q} \\
\dot{n} \\
\end{array}$ & 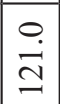 & $\dot{\hat{\sigma}}$ & 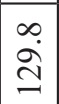 & $\vec{\infty}$ & $\begin{array}{l}\vec{\sim} \\
\ddot{\infty}\end{array}$ \\
\hline & 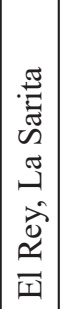 & 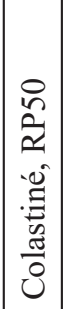 & 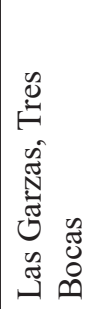 & 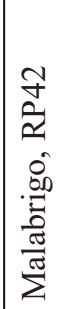 & 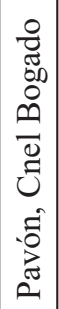 & 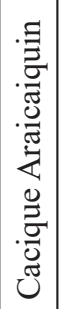 & 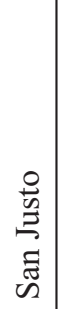 & $\begin{array}{l}\frac{0}{0} \\
0 \\
0 \\
\sum_{0}^{0} \\
. \frac{\pi}{3} \\
\frac{0}{0} \\
ن\end{array}$ & 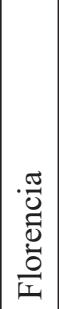 & 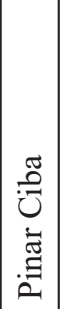 & 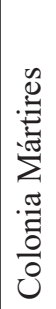 & 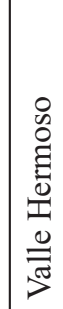 & 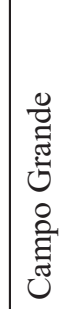 & 樆 & 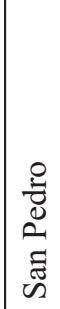 & 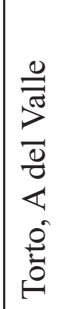 & 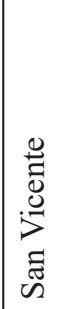 & 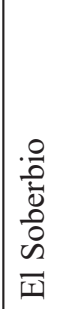 & 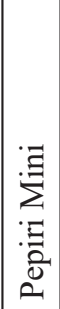 & 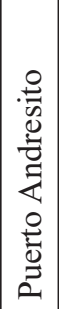 & 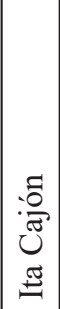 & $\mid \begin{array}{c}0 \\
0 \\
0 \\
\Xi \\
0 \\
0 \\
\tilde{D} \\
\tilde{2}\end{array}$ & 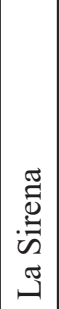 & 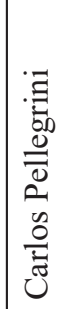 & 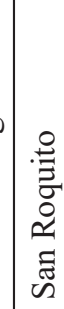 \\
\hline
\end{tabular}




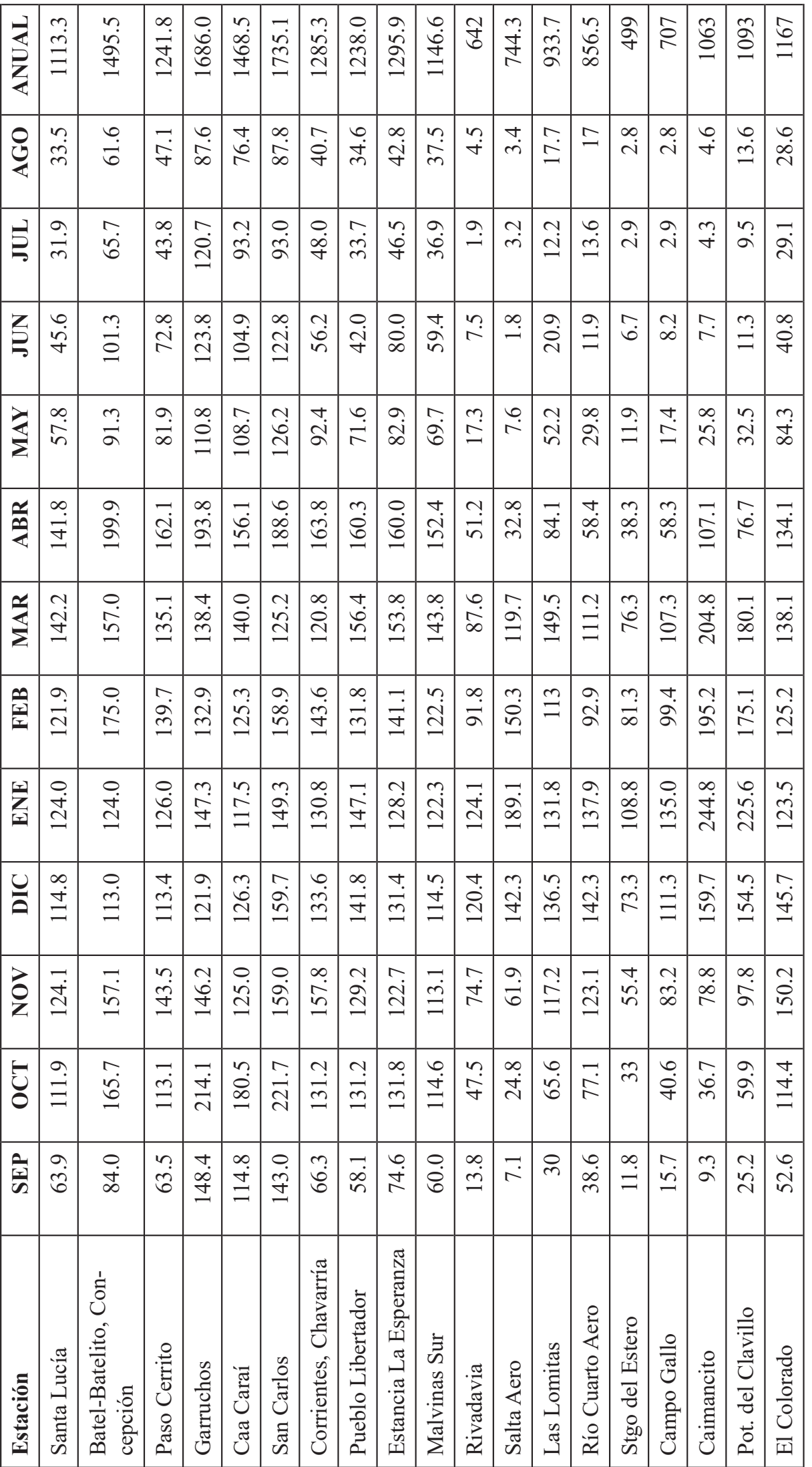




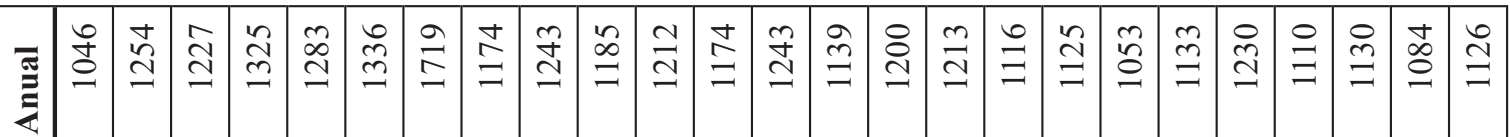

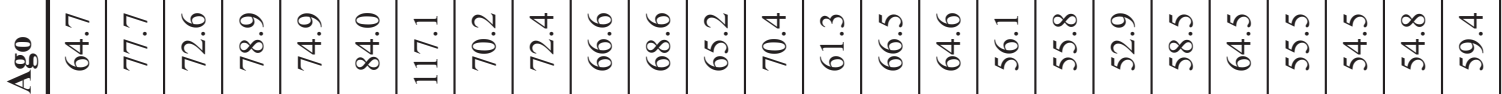

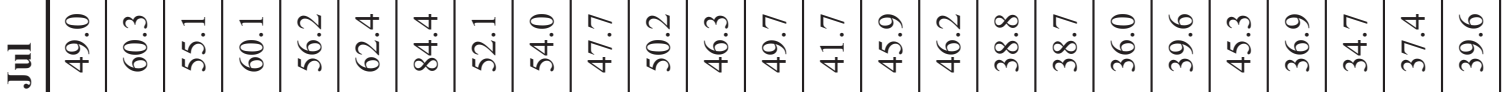

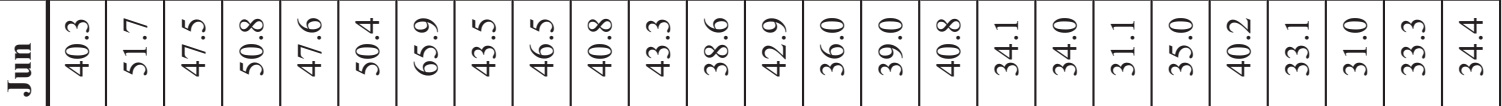

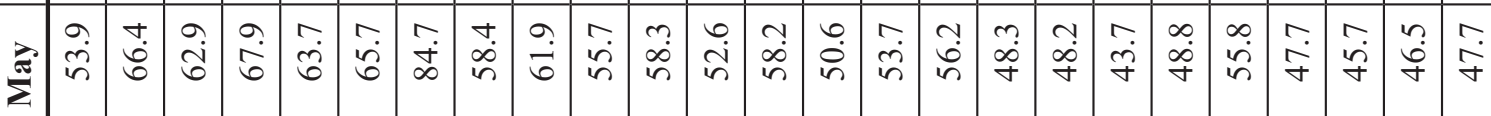

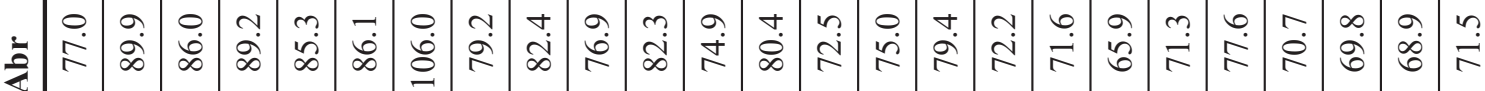

- m

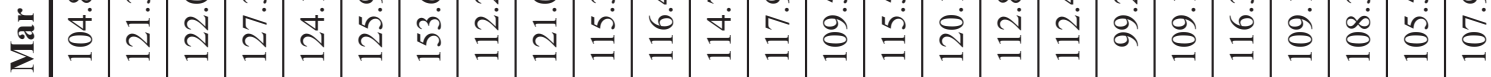

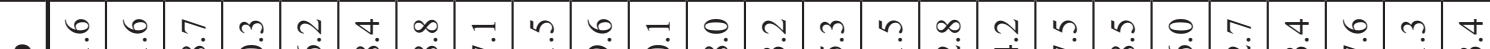

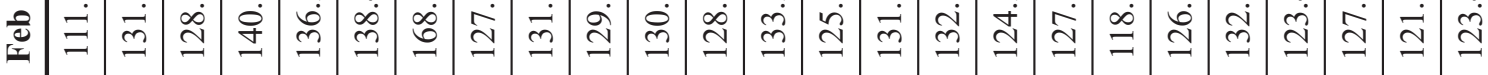
-

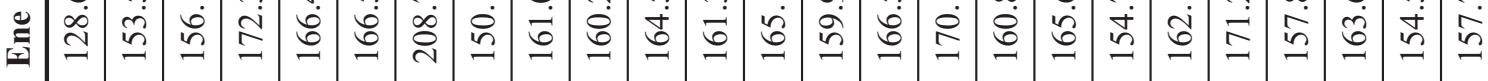

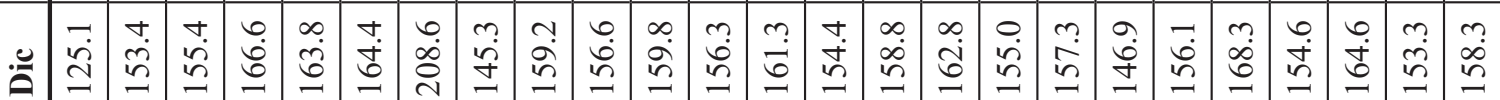

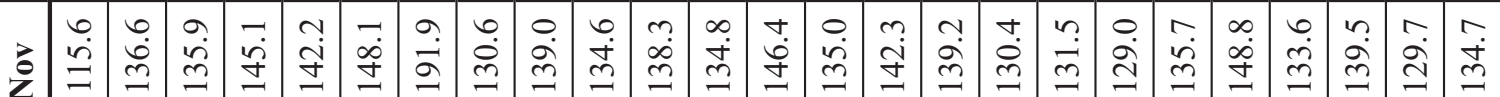

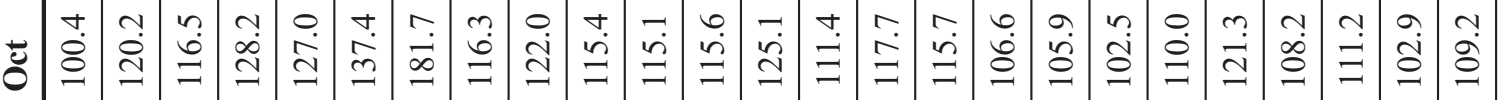

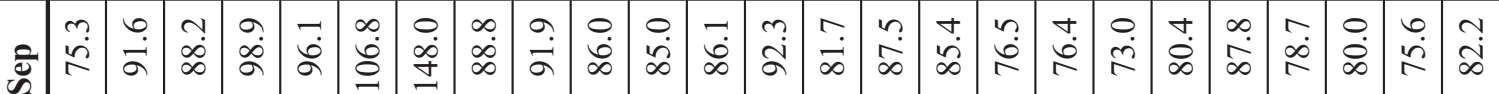

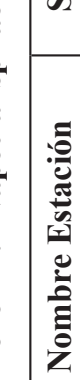

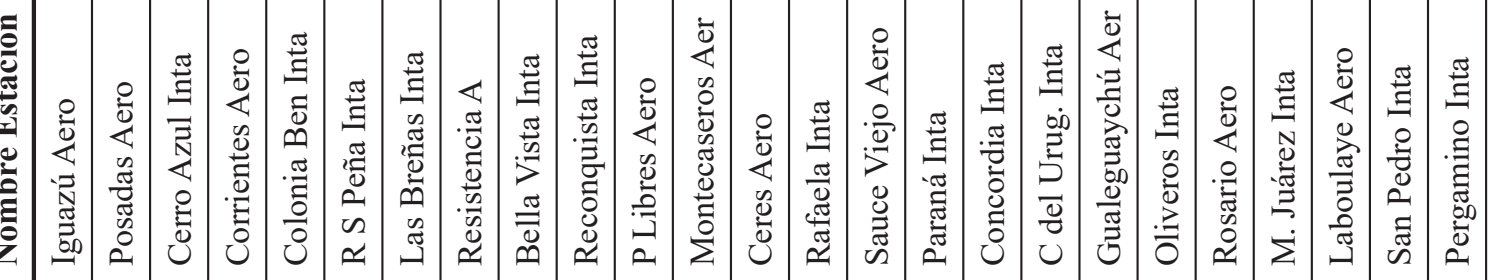




\begin{tabular}{|c|c|c|c|c|c|c|c|c|c|c|c|c|c|}
\hline $\bar{\Xi}$ & $\stackrel{\infty}{\infty}$ & 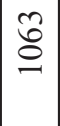 & 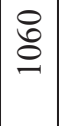 & $\stackrel{8}{\&}$ & $\stackrel{\circ}{\stackrel{2}{=}}$ & a & ๙ે & $\hat{\delta}$ & 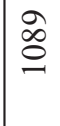 & $\begin{array}{l}\infty \\
\infty\end{array}$ & 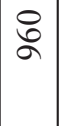 & $\hat{\circ}$ & ळे \\
\hline$\stackrel{80}{4}$ & $\begin{array}{l} \\
\dot{+} \\
\dot{n}\end{array}$ & \begin{tabular}{|c|}
0 \\
$\dot{n}$ \\
$n$
\end{tabular} & \begin{tabular}{|l|}
\multirow{\sigma}{\sigma}{} \\
\end{tabular} & $\frac{y}{n}$ & $\underset{n}{i n}$ & oे & 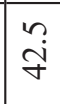 & $\begin{array}{l}0 \\
\dot{\sigma} \\
\end{array}$ & $\stackrel{n}{i}$ & $\frac{\dot{x}}{n}$ & $\stackrel{\vec{i}}{\vec{f}}$ & $\begin{array}{l}0 \\
\dot{m}\end{array}$ & $\begin{array}{l}\dot{\rho} \\
\dot{m}\end{array}$ \\
\hline $\bar{\Xi}$ & $\begin{array}{l}\hat{m} \\
\dot{m}\end{array}$ & $\vec{m}$ & $\stackrel{m}{m}$ & $\begin{array}{l}\stackrel{Y}{*} \\
\stackrel{m}{m}\end{array}$ & m & 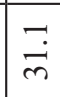 & $\hat{\grave{\lambda}}$ & $\frac{0}{\dot{m}}$ & $\stackrel{n}{m}$ & $\frac{n}{m}$ & $\vec{b}$ & 六 & $\mid \begin{array}{l}\infty \\
\dot{\sim}\end{array}$ \\
\hline$\Xi$ & $\stackrel{+}{\dot{m}}$ & \begin{tabular}{|l|}
$\infty$ \\
$\dot{\lambda}$ \\
$\dot{\lambda}$
\end{tabular} & $\begin{array}{l}n \\
\dot{n} \\
\dot{m}\end{array}$ & $\begin{array}{l}\infty \\
\dot{m} \\
\dot{m}\end{array}$ & $\frac{n}{m}$ & $\vec{\infty}$ & 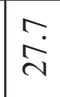 & $\overrightarrow{\dot{m}}$ & $\begin{array}{l}n \\
0 \\
0 \\
n\end{array}$ & $\begin{array}{l}0 \\
\dot{m} \\
m\end{array}$ & $\begin{array}{l}n \\
\tilde{n} \\
\end{array}$ & $\stackrel{+}{\vec{\lambda}}$ & $\hat{\mathrm{i}}$ \\
\hline$\vec{J}$ & $\begin{array}{l}\dot{0} \\
\dot{\gamma}\end{array}$ & $\begin{array}{l}\dot{q} \\
\dot{\gamma}\end{array}$ & $\begin{array}{l}\tilde{y} \\
\tilde{\gamma}\end{array}$ & $\begin{array}{l}\dot{\sim} \\
\dot{q}\end{array}$ & $\frac{m}{n}$ & $\mid \begin{array}{l}\infty \\
\dot{q}\end{array}$ & $\stackrel{\circ}{\dot{m}}$ & $\overline{\check{g}}$ & $\begin{array}{l}\infty \\
\dot{\gamma}\end{array}$ & $\begin{array}{l}\dot{J} \\
\dot{f}\end{array}$ & $\begin{array}{l}n \\
\dot{m} \\
\dot{m}\end{array}$ & $\begin{array}{l}\infty \\
\dot{m} \\
m\end{array}$ & $\underset{\dot{m}}{\stackrel{\circ}{\dot{m}}}$ \\
\hline$\frac{3}{4}$ & 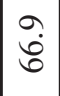 & $\frac{n}{6}$ & $\dot{8}$ & $\begin{array}{l}\dot{\theta} \\
\dot{\theta}\end{array}$ & 竎 & ळे & ڤें & $\begin{array}{l}+ \\
\dot{J}\end{array}$ & 它 & बें & $\begin{array}{l}+ \\
\infty \\
i \\
i\end{array}$ & $\frac{0}{i n}$ & $\begin{array}{l}\overrightarrow{0} \\
\text { in }\end{array}$ \\
\hline$\sum$ & $\begin{array}{l}m \\
\tilde{0} \\
0\end{array}$ & $\begin{array}{l}n \\
i \\
0 \\
0\end{array}$ & $\begin{array}{l}0 \\
\dot{a} \\
0\end{array}$ & 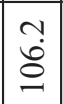 & $\stackrel{t}{\stackrel{t}{\Xi}}$ & $\stackrel{9}{0}$ & $\begin{array}{l}m \\
\infty \\
\infty \\
\infty\end{array}$ & $\begin{array}{l}n \\
\stackrel{2}{0} \\
\end{array}$ & \begin{tabular}{|l|} 
\\
$\dot{0}$ \\
$\dot{0}$
\end{tabular} & $\begin{array}{l}\infty \\
\dot{J}\end{array}$ & $\hat{a}$ & $\frac{a}{a}$ & กู \\
\hline 巳 & \begin{tabular}{|c|} 
\\
$\stackrel{\Xi}{\Xi}$ \\
\end{tabular} & $\overrightarrow{\tilde{d}}$ & $\begin{array}{l}\infty \\
\mathbb{i} \\
\mathbb{Z}\end{array}$ & $\mid \stackrel{?}{\stackrel{I}{I}}$ & $\begin{array}{l}\infty \\
\dot{n} \\
\tilde{n}\end{array}$ & $\begin{array}{l}0 \\
\stackrel{1}{1}\end{array}$ & $\stackrel{n}{\Xi}$ & $\begin{array}{l}\infty \\
\infty \\
\stackrel{\infty}{=}\end{array}$ & 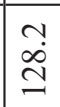 & $\stackrel{?}{\stackrel{n}{\Xi}}$ & $\begin{array}{l}\infty \\
\infty \\
\stackrel{\infty}{=} \\
\end{array}$ & $\stackrel{\check{m}}{=}$ & $\stackrel{?}{\Xi}$ \\
\hline$\stackrel{\Xi}{=}$ & \begin{tabular}{|l|}
$n$ \\
$\infty$ \\
$n$ \\
$n$
\end{tabular} & $\vec{n}$ & 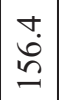 & $\overrightarrow{\mathrm{d}}$ & $\underset{+}{\stackrel{+}{I}}$ & $\begin{array}{l}m \\
n \\
n\end{array}$ & $\begin{array}{l}\hat{\sigma} \\
\dot{m} \\
\end{array}$ & 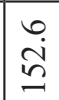 & & $\begin{array}{l}\hat{i} \\
\dot{I}\end{array}$ & $\begin{array}{l}\stackrel{+}{\dot{a}} \\
\dot{g}\end{array}$ & 妾 & 宇 \\
\hline 光 & 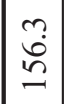 & $\begin{array}{l}\vec{i} \\
\stackrel{2}{n}\end{array}$ & $\begin{array}{l}0 \\
\dot{+} \\
\end{array}$ & $\begin{array}{l}r \\
\text { in } \\
n\end{array}$ & $\begin{array}{l}\infty \\
\dot{\mathbb{d}} \\
\end{array}$ & $\begin{array}{l}? \\
\text { 守 } \\
\end{array}$ & $\begin{array}{l}0 \\
\dot{0} \\
\dot{m}\end{array}$ & $\begin{array}{l} \\
\dot{J} \\
\end{array}$ & $\begin{array}{l}a \\
\infty \\
0\end{array}$ & \begin{tabular}{|l|}
\multirow{2}{*}{} \\
$\stackrel{\text { D }}{ }$
\end{tabular} & \begin{tabular}{|l|}
$\dot{g}$ \\
$\dot{g}$
\end{tabular} & $\frac{\infty}{m}$ & $\stackrel{\sim}{\sim}$ \\
\hline ż & $\begin{array}{l}m \\
\stackrel{m}{2} \\
\stackrel{2}{2}\end{array}$ & 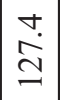 & 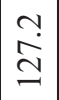 & $\left|\begin{array}{l}\infty \\
\grave{\beth} \\
\grave{\beth}\end{array}\right|$ & $\begin{array}{l}\hat{n} \\
\ddot{n}\end{array}$ & ?̊. & \begin{tabular}{|l|}
0 \\
0 \\
$\stackrel{\infty}{0}$
\end{tabular} & ڤે & 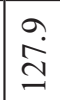 & $\stackrel{0}{\ominus}$ & $\stackrel{m}{n}$ & 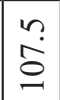 & 官 \\
\hline$\ddot{0}$ & \begin{tabular}{|c|}
0 \\
$\dot{0}$ \\
0
\end{tabular} & $\begin{array}{l}n \\
8 \\
0\end{array}$ & 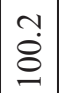 & $\begin{array}{l}n \\
\tilde{O} \\
\end{array}$ & $\begin{array}{l}m \\
\infty \\
\infty\end{array}$ & 广̃ & $\vec{\infty}$ & $\frac{1}{\sigma}$ & $\begin{array}{l}\infty \\
\dot{\alpha}\end{array}$ & \begin{tabular}{l|}
0 \\
$\dot{\alpha}$ \\
\end{tabular} & $\begin{array}{l} \\
\dot{8}\end{array}$ & $\begin{array}{c}m \\
\infty \\
\infty\end{array}$ & $\frac{\sigma}{\infty}$ \\
\hline हूँ & $\begin{array}{l}0 \\
\dot{0} \\
i\end{array}$ & 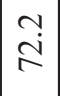 & $\begin{array}{l}\infty \\
\dot{R} \\
\end{array}$ & $\begin{array}{l}N \\
\\
\end{array}$ & $\stackrel{0}{2}$ & 审 & $\begin{array}{l}n \\
\text { in }\end{array}$ & $\hat{\sigma}$ & $\begin{array}{l}\infty \\
i \\
\mathbb{i}\end{array}$ & वें & $\begin{array}{l}\mathrm{N} \\
\mathrm{i} \\
\end{array}$ & $\hat{D}$ & $\hat{s}$ \\
\hline 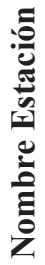 & 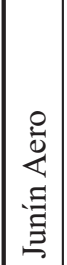 & 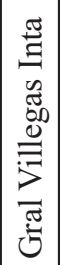 & 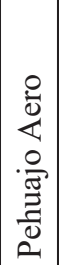 & 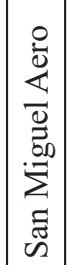 & 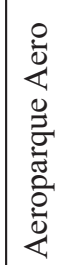 & 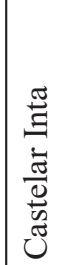 & 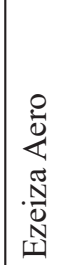 & 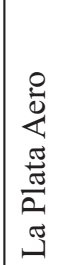 & 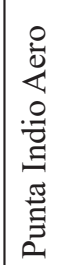 & $\mid \begin{array}{l}0 \\
0 \\
0 \\
0 \\
0 \\
0 \\
0 \\
0 \\
0 \\
0\end{array}$ & 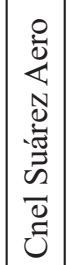 & 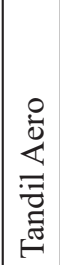 & 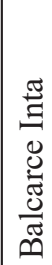 \\
\hline
\end{tabular}

